\title{
CARACTERIZACIÓN DE LA CIMENTACIÓN Y SUELO SUBYACENTE DE LA GIRALDA DE SEVILLA/ESPAÑA
}

\author{
(CHARACTERIZATION OF ITS FOUNDATION AND UNDERLYING SOIL OF THE GIRALDA'S OF \\ SEVILLA/SPAIN)
}

Ángela Barrios Padura, Prof. Asociado E.T.S. Arquitectura de Sevilla; Ignacio Valverde Espinosa, C.E.U. Arquitectura de Sevilla; Jorge Polo Velasco, Prof. Titular E.U. Arquitectura Técnica de Sevilla; Miguel A. Fernández Vélez y Ángel Martinez Girón, VORSEVI, S.A.

Dirección: Alfonso Jiménez y Jesús Barrios Sevilla, C.U.E.T.S. Arquitectura de Sevilla

ESPAÑA

Fecha de recepción: $30-X-97$

\section{RESUMEN}

Este estudio se ha realizado con el objetivo de investigar la naturaleza, tipologia y composición de la cimentación de la Giralda, asi como las caracteristicas del terreno subyacente que sirve de apoyo, coincidiendo con la celebración de los 800 años de la terminación de la torre musulmana. Los resultados obtenidos han puesto de manifiesto aspectos tan importantes como la profundidad de la cimentación, en torno a $5 \mathrm{~m}$ desde la actual cota de acerado; la existencia de un pequeño sobreancho en la misma; su composición, a base de sillares de piedra calcarenita; la preparación del terreno de apoyo... Por lo que respecta al suelo, se ha determinado su capacidad portante; los asientos producidos en las diferentes fases de la construcción de la torre y su evolución en el tiempo; el asiento diferencial que justifica la muy ligera inclinación que presenta.

\section{SUMMARY}

The objetive of this work is to investigate the Giralda's typology and composition of its foundation, as well as the characteristics of the underlying foundation soil, coincident with the celebration of the 800 th anniversary of completing the muslin tower. The results of the investigation show us some important aspects as foundation deep, around 5 meters from the actual pavement; the existence of a small overwide in it; its composition made of calcarenite bedstone; the preparation of the support... In relation to the soil it has been fixed its bearing capacity; the abasements along the different phases in the tower's building and its time evolution; the differential abasement that justify the very small heeling and other soil characteristics, that explain the stability of this Universal Monument.

\section{Antecedentes}

En este trabajo se ha pretendido integrar los datos aportados por las diferentes campañas geotécnicas, basadas en sondeos rotatorios, con recuperación continua de testigo y realizadas junto a la Giralda (ver Fig. 1), que reflejan la situación de: (12 sondeos junto a las fachadas Norte, Este y Sur -4 de ellos, inéditos- (1) y otros 4 más en las

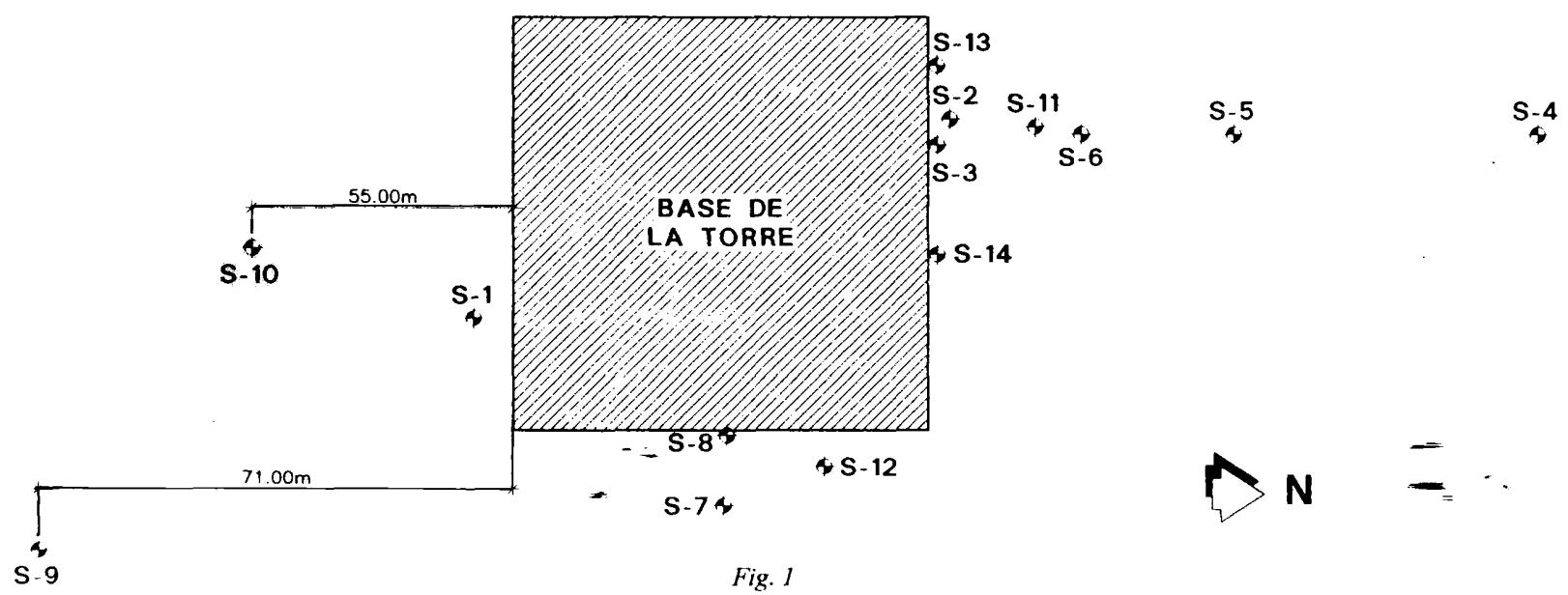


inmediaciones, ligeramente desplazados al Sur-Suroeste); los ensayos realizados en laboratorio con las muestras obtenidas y las diferentes hipótesis hasta ahora barajadas. Se ha intentado, pues, clarificar, en la medida de lo posible, la disposición del cimiento, su profundidad, sección, etc.

El análisis efectuado ha permitido un estudio más amplio sobre el asiento inducido por la torre, la evolución temporal en función de la carga transmitida y realizar una breve discusión sobre el origen de la desviación que presenta $\left(0.12^{\circ}\right.$ hacia el SE).

Gran parte de los cálculos realizados se apoyan en los datos que se recogen en el libro "Tvrris Fortissima. Documentos sobre la Construcción, Acrecentamientoy Restauración de la Giralda", del año 1988 y "Estudio de la cimentación y terreno subyacente a la Torre de la Giralda de la Catedral de Sevilla", febrero de 1998, realizado por VORSEVI, S.A., trabajos ambos realizados por parte del equipo de investigación que redacta este trabajo.

\section{Caracterización de los suelos de apoyo. Estratigrafía}

La zona estudiada se asienta sobre sedimentos fluviales del Guadalquivir y de arroyos afluentes, cuyo espesor supera los $18 \mathrm{~m}$ bajo el pie de la torre.

Subyacente y discordante con este paquete sedimentario afloran las arcillas, más o menos margosas, grisáceas o verdosas ("margas azules"), de ambiente claramente marino y espesor de varios cientos de metros, que han sido datadas como Mioceno.
Se describen a continuación y de forma muy abreviada, los aspectos geotécnicos y estratigráficos esenciales de los términos que componen el paquete fluvial, el sustrato y la eventual disposición de rellenos tapizando todo el conjunto. Los valores medios deducidos aquí serán empleados posteriormente en los cálculos y se han incluido, de forma aproximada, en el perfil Norte-Sur de la figura 2.

Desde la posición más superficial a la más profunda, tenemos los siguientes términos:

\subsection{Cimentación}

Los cimientos se reconocieron en los seis sondeos realizados junto a la torre, obteniéndose las siguientes profundidades relacionadas con la distancia a la cara vertical en que se inicia la perforación (Tabla 1).

De acuerdo con estos datos y con el hecho de que los demás sondeos realizados en los alrededores, hasta una distancia de la torre de $1.5 \mathrm{~m}$ no cortan el cimiento, puede deducirse que el sobreancho máximo de la cimentación se sitúa en torno a ese valor de $1.5 \mathrm{~m}(2)$.

Dado que la parte emergida presenta una sección cuadrada de $13.61 \mathrm{my}$, teniendo en cuenta el sobreancho, se confirma que el plano de apoyo es un cuadrado de $15.11 \mathrm{~m}$ de lado. Se ha simplificado este valor a $15.0 \mathrm{~m}$ en los cálculos, ya que parece más apropiado, por la precisión del dato.

El escalonamiento de este cimiento no queda bien definido

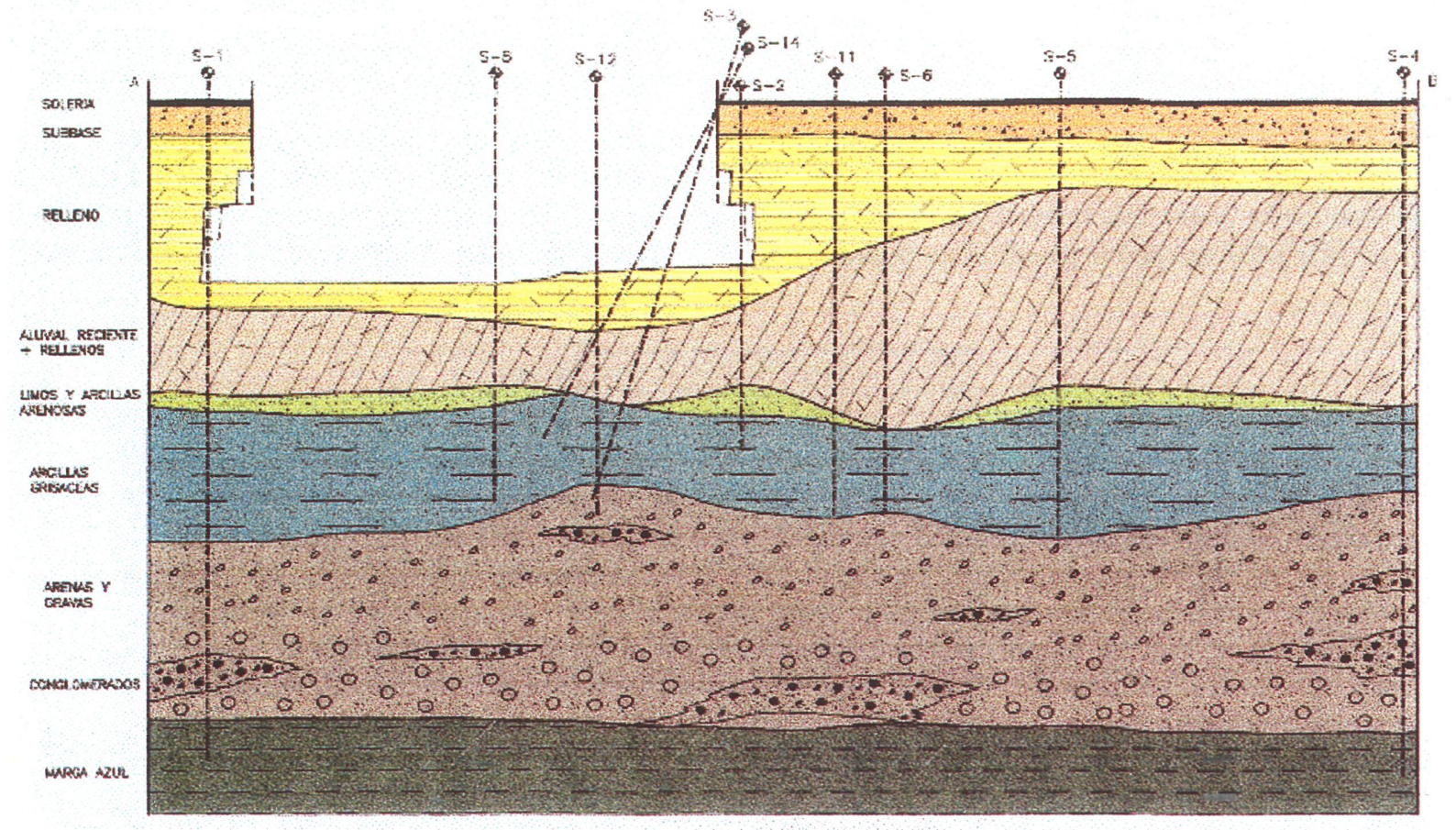


TABLA 1

\begin{tabular}{|c|c|c|c|}
\hline Sondeo & Distaneia (n) & Cota cimiento (m) & Espesor reconocido $(\mathrm{m})$ \\
\hline$S-1$ & 1.5 & $4.00-6.00$ & 2.00 \\
\hline$S-2$ & 0.8 & $3.00-6.00$ & 3.00 \\
\hline$S-3$ & 0 & $0.00-5.20$ & 5.20 \\
\hline$S-8$ & 0 & $0.00-5.30$ & 5.30 \\
\hline$S-13$ & 0 & $0.00-5.00$ & 5.00 \\
\hline$S-14$ & 0 & $0.00-4.83$ & 4.83 \\
\hline
\end{tabular}

con los sondeos realizados, aunque prácticamente puede asegurarse que el ancho de la base de la cimentación se sitúa a unos $3 \mathrm{~m}$ de profundidad (S-2). A esa cota, la cimentación está conformada por un lecho más amplio, que confirma una base de apoyo, constituida por un cuadrado de unos $15 \mathrm{~m}$ de lado. Tomando como base los datos históricos y la investigación efectuada, el proceso seguido en la ejecución de la cimentación comprende los siguientes aspectos:

1. La excavación de la plataforma de cimentación, hasta una profundidad entre los 5 y $8 \mathrm{~m}$ desde la superficie actual. Labores que pueden realizarse con rapidez dada la naturaleza de los materiales, esencialmente aluviones y rellenos de baja consistenciay compacidad. El talud se realizó con una inclinación de unos $45^{\circ}$ a $60^{\circ}$, con un posible escalonamiento a la cota de $-3 \mathrm{~m}$.

2. Previsiblemente, el nivel freático en la zona se disponía sensiblemente por debajo de su posición actual, constituyendo un acuífero colgado sobre el nivel regional correspondiente al del Guadalquivir. En este sentido, conviene señalar que:

- El cauce del río no coincidía con el actual, presentando diversos ramales tributarios.

- Existe referencia que durante la excavación aparece un "pozo manantial" que debió cegarse para continuar las obras. Puediendo asegurarse, por tanto, que la excavación se sitúa por debajo del nivel freático primitivo (situación explicable por la baja transmisividad de los niveles limo arcillosos del nivel II) y que, ante la imposibilidad de continuar la excavación, se procedió a rellenar la zona con todo tipo de material (restos cerámicos, sillares de areniscas, ladrillos, etc.) cubriéndolos y enrasándolos con mortero de cal, hasta conseguir una explanación a la que referir el plano del cimiento. Este relleno alcanza una mayor profundidad en la cara este de la torre.

- La capa más superficial de relleno, de aproximadamente $2 \mathrm{~m}$ de espesor, puede haberse vertido sobre la superficie topográfica preexistente, por lo que la excavación habria que referirla a esta cota. Recordemos, además, que hacia el Oeste, la topografia baja sensiblemente (al menos $1-1.5 \mathrm{~m}$ en la actualidad).

3. El cimiento se construye con rapidez tras la excavación, empleándose aproximadamente 5 años entre ambas labores, facilitadas porque el material empleado se obtiene de la muralla romana próxima, según se detalla en la descripción histórica del proceso constructivo.

4. La disposición del cimiento se corresponde con hileras de sillares de areniscas recibidos con mortero, alternantes, con niveles de hormigón calizo de regularización y ladrillos.

En resumen, podemos concluir que el cimiento tiene una profundidad media máxima de unos $5.1 \mathrm{~m}$, con una sección a esta cota de $225 \mathrm{~m}^{2}$ y está constituido por sillares de piedra arenisca con mortero de cal, alternando con conglomerados de gravas y/o cerámica con mortero de cal, en espesores y zonas variables. Esta cimentación apoya sobre una explanada mejorada de espesor variable (entre 2 y $4 \mathrm{~m}$, o incluso más) constituida por todo tipo de material cerámico, ladrillos, fragmentos de areniscas, etc., conglomerados con mortero calizo de diversa calidad y posibles restos romanos.

\subsection{Estratigrafia (Fig. 3). ,}

\subsubsection{Solería y rellenos recientes}

Se trata de un nivel que agrupa varias capas sucesivas de rellenos, de litología diversa e irregular distribución.

En general, bajo la capa de solería y hormigón de los aledaños de la torre, se encuentra una subbase arenosa con gravas de tonalidad marrón, correspondiente al relleno más reciente (localizándose, aquí, el mayor número de ensayos).

Bajo este nivel aparece un entramado de estratos esencialmente limosos y arcillosos, aunque con contenido muy variable en arenas y gravas, restos cērámicos y materia orgánica, que se extienden hasta casi $4 \mathrm{~m}$ de profundidad. No obstante, se ha situado este nivel por encima del nivel freático medio de la zona. 


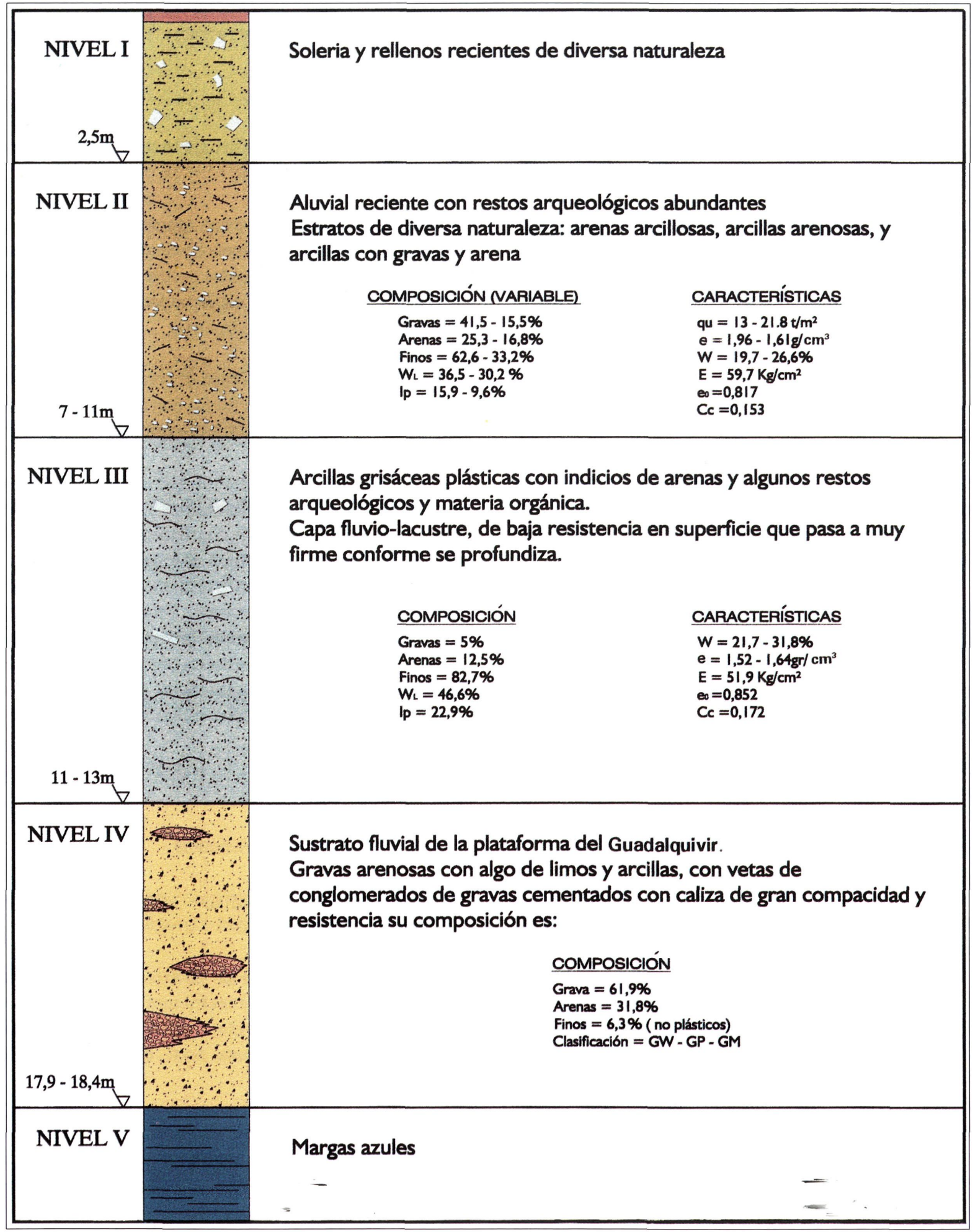


Los sondeos reconocen este nivel entre las distintas cotas (Tabla 2).

Los principales parámetros geotécnicos se resumen a continuación:

\section{GRANULOMETRÍA}

Los análisis granulométricos por tamizado muestran un predominio de la fracción grava y restos cerámicos sobre los tramos arenosos y con finos (Tabla 3).

\section{PLASTICIDAD}

Los finos resultaron de baja o nula plasticidad, oscilando entre diferentes valores (Tabla 4).

De acuerdo con estos datos el suelo se clasifica como gravas arenosas con abundantes restos cerámicos y con arcillas y limos (más abundantes en profundidad): GM y GM-GC.

\section{COMPACIDAD}

De acuerdo con los datos registrados en la perforación y por su naturaleza, podemos asignar a este nivel una compacidad baja o muy baja, a pesar de no disponer de ensayos.

\subsubsection{Aluvial reciente - Rellenos antiguos}

Se ha incluido en este nivel una amplia franja de terreno de atribución incierta, que alterna los rellenos antrópicos con depósitos fluviales, también recientes, dado que incluyen gran cantidad de restos cerámicos.

Por su litología y posición estratigráfica pueden diferenciarse varios subniveles:

- Subnivel 3A. Arenas arcillosas marrones con restos cerámicos: $\mathrm{GC}, \mathrm{SC}$.

- Granulométricamente, tenemos:

Grava: $41.5 \%(29-55)$

Arena: $25.3 \%(15-39)$

Finos: $33.2 \%(24-44)$

- Los finos son poco plásticos:

$$
\begin{aligned}
& \mathrm{W}_{\mathrm{L}}=30.2(27.5-31.3) \\
& \mathrm{W}_{\mathrm{P}}=20.6(19.7-21.7) \\
& \mathrm{I}_{\mathrm{P}}=9.6(7.2-10.9)
\end{aligned}
$$

- Presenta un contenido significativo en materia orgáni-

\begin{tabular}{|c|c|c|}
\hline Sondeo & Cota aproxnmoda relleno (n) & Espesor reconocido (n) \\
\hline$S-1$ & $0.00-4.00$ & 4.00 \\
\hline$S-2$ & $0.00-3.00$ & 3.00 \\
\hline S-4 & $0.00-2.60$ & 2.60 \\
\hline$S-5$ & $0.00-2.50$ & 2.50 \\
\hline S-6 & $0.00-2.90$ & 2.90 \\
\hline S-7 & $0.00-2.80$ & 2.80 \\
\hline$S-11$ & $0.00-3.00$ & 3.00 \\
\hline$S-12$ & $0.00-4.60$ & +60 \\
\hline
\end{tabular}
ca: $1.3-2.1 \%$ (proximidad a una cloaca).

TABLA 2

TABLA 3

\begin{tabular}{|c|c|c|c|}
\hline Parametro & Rang o de yartacion & \multicolumn{2}{|c|}{ Anornedo } \\
\hline L.mite licuido & NP-38.9 & 19.8 & $=$ \\
\hline Limute plástico & $N P-31.6$ & 15.2 & - \\
\hline Indice plasticidad & $N P-10.8$ & 4.6 & \\
\hline
\end{tabular}

\begin{tabular}{|c|c|c|}
\hline Fracción & Rango de Vrarión \% & Valor medio \\
\hline Grava & $37-84$ & 54.3 \\
\hline Arena & $7-36$ & 28 \\
\hline Finos & $4-33$ & 17.7 \\
\hline
\end{tabular}

TABLA 4 
. Subnivel 3B. Arcillas arenosas marrón grisáceas a pardas: CL.

- Granulometría:

Grava: $15.5(4-29)$

Arena: $21.9(18-26)$

Finos: $62.6(46-78)$

- Los finos resultaron esencialmente arcillosos, de plasticidad media:

$$
\begin{aligned}
& \mathrm{W}_{\mathrm{L}}=36.5(27.3-42.6) \\
& \mathrm{W}_{\mathrm{p}}=20.6(19.6-22.1) \\
& \mathrm{I}_{\mathrm{P}}=15.9(7.2-20.5)
\end{aligned}
$$

- La cohesión resulta anormalmente elevada:

$$
\begin{aligned}
& C^{\prime}=3.0 \mathrm{t} / \mathrm{m}^{2} \\
& \theta^{\prime}=5^{\circ}
\end{aligned}
$$

- La densidad seca es de $1.61 \mathrm{t} / \mathrm{m}^{3}$ y su humedad $\mathrm{w}=19.7 \%$.

- Presenta consistencia FIRME, $\mathrm{q}_{\mathrm{u}}=13 \mathrm{t} / \mathrm{m}^{2}$

- El contenido en materia orgánica oscila entre un 0.4 y $1.4 \%$.

- No se detecta presencia de sulfatos en este nivel.

. Subnivel 3C. Arcillas con grava, arena y restos cerámicos esporádicos $\mathrm{CL}, \mathrm{GC}, \mathrm{CH}$.

- Granulometría:

Grava: $26.6 \%(0-46)$

Arena: $16.8 \%(0.5-38)$

Finos: $56.6 \%(23-99.5)$

- Plasticidad:

$$
\begin{gathered}
\mathrm{W}_{\mathrm{L}}=35.9(29.1-56.5) \\
\mathrm{W}_{\mathrm{P}}=22.6(18.9-29.3) \\
\mathrm{I}_{\mathrm{P}}=13.3(8.9-27.2)
\end{gathered}
$$

- La densidad aparente oscila entre 1.93 y $1.99 \mathrm{t} / \mathrm{m}^{3}$ con $w=31$ y 26.6 respectivamente.

\section{- Presenta consistencia MUY FIRME, $\mathrm{q}_{\mathrm{u}}=21.8 \mathrm{t} / \mathrm{m}^{2}$}

- El contenido en materia orgánica oscila entre el 1.1 y $8.9 \%$ en peso (proximidad a una cloaca).

- Los sulfatos oscilaron entre $0-0.5 \%$. -

Se puede afirmar que se trata de un nivel irregular y discontinuo, con espesor muy variable, aunque con cierta homogeneidad en sus principales parámetros geotécnicos Se ha situado este nivel entre las cotas que se indican en la tabla 5.

Dado que para el cálculo de asientos se considera esta capa como única, se han agrupado los ensayos edométricos realizados, obteniéndose los siguientes valores medios:
Módulo edométrico Índice de poros inicial Índice de compresión

$$
\begin{aligned}
& \mathrm{E}_{\mathrm{m}}=59.7 \mathrm{kp} / \mathrm{cm}^{2}(52.2-66) \\
& \mathrm{e}_{0}=0.817(0.754-0.827) \\
& \mathrm{C}_{\mathrm{c}}=0.153(0.12-0.17)
\end{aligned}
$$

2.2.3. Arcillas grisáceas plásticas, con indicios de arena y restos cerámicos.

Esta capa de origen fluvio-lacustre aparece frecuentemente a techo del nivel de gravas, marcando el tránsito hacia una sedimentación fluvial más potente.

Se trata de margas de tonalidad grisácea, de mediana plasticidad, con textura masivay con consistencia variable, a techo presentan consistencia fangosa (a veces resulta dificil de delimitar con claridad esta capa del relleno suprayacente), que rápidamente aumenta en profundidad, hasta presentar una apariencia de firme a muy firme.

Este nivel se ha reconocido en los sondeos entre las cotas que pueden verse en la tabla 6.

A continuación se resumen los principales parámetros analizados en el seno de este nivel:

\section{GRANULOMETRÍA}

Los análisis granulométricos por tamizado muestran un contenido en finos claramente mayoritario, contaminado en la zona de techo por tramos arenosos y con restos cerámicos $(4 \mathrm{~A})$ (Tabla 7$)$.

\section{PLASTICIDAD}

Salvo en la zona superior y en tramos minoritarios este estrato presenta plasticidad media alta (Tabla 8).

De acuerdo con estos datos el suelo se clasifica como $\mathrm{CH}$, CL y, puntualmente, SM.

\section{HUMEDAD NATURAL}

La humedad natural oscila entre un 21.7 y un $31.8 \%$, manteniéndose en valores bastante constantes, ligeramente por encima del límite plástico.

\section{DENSIDAD APARENTE -}

La densidad seca varía entre 1.52-1.64 (1.57) $\mathrm{t} / \mathrm{m}^{3}$

La densidad húmeda varía entre 1.89-2.12 (1.96) t/ $\mathrm{m}^{3}$. 
TABLA 5

\begin{tabular}{|c|c|c|}
\hline Sondeo & Cota aproximada gluvial relleno (m) & Espesor reconocido (m) \\
\hline$S-1$ & $6.00-9.00$ & 3.00 \\
\hline$S-2$ & $6.00-8.20$ & 2.20 \\
\hline$S-3$ & $5.20-9.30$ & 4.10 \\
\hline$S-4$ & $2.60-8.80$ & 6.20 \\
\hline$S-5$ & $2.50-10.80$ & 8.30 \\
\hline$S-6$ & $2.90-9.50$ & 6.60 \\
\hline S-7 & $2.80-11.20$ & 8.40 \\
\hline S-8 & $5.30-10.80$ & 5.50 \\
\hline$S-9$ & $5.60-7.40$ & 1.80 \\
\hline$S-10$ & $5.40-7.60$ & 2.20 \\
\hline$S-11$ & $3.00-7.00$ & 4.00 \\
\hline $\mathrm{S}-12$ & $4.60-8.00$ & 3.40 \\
\hline$S-13$ & $5.00-7.16$ & $>2.16$ \\
\hline$S-14$ & $8.49-9.84$ & $>1.35$ \\
\hline
\end{tabular}

TABLA 6

\begin{tabular}{|c|c|c|}
\hline Sondeo & Cota aproximada aluvial relleno (m) & Espesor reconocido (11) \\
\hline$S-1$ & $9.00-12.80$ & 3.80 \\
\hline$S-2$ & $8.20-10.10$ & $>1.90$ \\
\hline$S-3$ & $9.30-11.60$ & 2.30 \\
\hline$S-4$ & $8.80-11.30$ & 2.50 \\
\hline S-5 & $10.80-12.70$ & 1.90 \\
\hline$S-6$ & $9.50-11.90$ & 2.40 \\
\hline$S-8$ & $10.80-11.60$ & 0.80 \\
\hline S-9 & $7.40-10.00$ & 2.60 \\
\hline$S-10$ & $7.60-11.20$ & 3.60 \\
\hline$S-11$ & $7.00-12.00$ & $>5.00$ \\
\hline$S-12$ & $8.00-11.15$ & 3.15 \\
\hline S-14 & $8.49-9.84$ & $>1.35$ \\
\hline
\end{tabular}

TABLA 7

\begin{tabular}{|c|c|c|}
\hline Eraccion & Rango de Variadion \% \% & Yalor medio \\
\hline Grava & $0-24$ & 5.05 \\
\hline Arena & $0.5-77$ & 12.25 \\
\hline Finos & $23-99.5$ & 82.7 \\
\hline
\end{tabular}

TABLA 8

\begin{tabular}{|c|c|c|}
\hline Parametro & Rongo do varacion & Volor medio \\
\hline Limite líquido & $26.3-64.5$ & 46.6 \\
\hline l.imite plástico & $17.2-30.8$ & 23.7 \\
\hline Indice plasticidad & $8.0-38.4$ & 22.9 \\
\hline
\end{tabular}

\section{CONSISTENCIA}

La consistencia es variable, prácticamente fangosa a techo y de muy firme a dura en la transición con las gravas arenosas subyacentes.

$$
\mathrm{q}_{\mathrm{u}}=2.3-55.6 \mathrm{t} / \mathrm{m}^{2}(23.2)
$$

(c) Consejo Superior de Investigaciones Científicas Licencia Creative Commons 3.0 España (by-nc)

\section{MÓDULO EDOMÉTRICO}

Los resultados obtenidos oscilaron entre:
Módulo edométrico
$\mathrm{E}_{\mathrm{m}}=51.9 \mathrm{kp} / \overline{\mathrm{cm}^{2}}(44.7-66)$
Índice de poros inicial
$\mathrm{e}_{0}=0.852(0.817-0.995)$
Índice de compresión
$\mathrm{C}_{\mathrm{c}}=0.172(0.166-0.183)$ 
Datos que se completan en los cálculos con otros ensayos edométricos realizados en sondeos próximos a la Giralda.

\section{COHESIÓN}

Se ha determinado la cohesión tanto en ensayos triaxiales como en ensayos de corte directo consolidado y drenado, obteniéndose los siguientes resultados:

Cohesión media $c^{\prime}=7 \mathrm{t} / \mathrm{m}^{2}$

Ángulo de rozamiento interno medio $\theta^{\prime}=10^{\circ}$.

\section{CONTENIDO EN MATERIA ORGÁNICA Y SULFA- TOS}

Todas las muestras de suelo presentan proporciones de materia orgánica variable, existiendo zonas en las que presumiblemente había un pozo negro o conducción de fecales, en las que el porcentaje llega al $18 \%$.

Por contra, los ensayos realizados no registran sulfatos formando parte del suelo.

\subsubsection{Gravas arenosas silíceas con algo de limos o arcilla} y con niveles conglomeráticos en la base

Este nivel constituye el sustrato de la serie fluvial de la plataforma del Guadalquivir en esta región y está representado por arenas y gravas arenosas marrones o verdosas, con cantos predominantemente silíceos, bien rodados, aunque de baja esfericidad.
Ocasionalmente, como parece ser el caso, encontramos niveles conglomeráticos discontinuos por cementación carbonática de estas gravas.

Este nivel ha sido reconocido en los sondeos entre las cotas indicadas en la tabla 9.

En base a la dificultad encontrada en su perforación, por la presencia de niveles conglomeráticos y por correlaciones con infinidad de sondeos en el mismo nivel en otros puntos de Sevilla, puede establecerse para esta capa una compacidad elevada, con módulos edométricos también elevados y asientos despreciables, de carácter instantáneo, por su elevada permeabilidad.

Los principales parámetros analizados se resumen a continuación (Tabla 10).

\section{GRANULOMETRÍA}

Los análisis granulométricos por tamizado muestran un acusado predominio de la fracción granular grosera, con lentejones arenosos de desigual proporción de finos (si bien, parte de los mismos, puede haberse lavado durante la perforación):

\section{PLASTICIDAD}

Los finos resultaron no plásticos o de plasticidad muy baja $\left(\mathrm{W}_{\mathrm{P}}=24.2, \mathrm{I}_{\mathrm{p}}=10.7\right)$. En base a ello el suelo se clasifica como gravas GW, GP o gravas limosas GM.

TABLA 9

\begin{tabular}{|c|c|c|}
\hline Sondeo & Cota aproximada aluvial-relleno $(\mathbf{m})$ & Espesor reconocido $(\mathbf{m})$ \\
\hline S-1 & $12.80-17.90$ & 5.10 \\
\hline S-3 & $11.60-12.00$ & $>0.40$ \\
\hline$S-4$ & $11.30-18.40$ & 7.10 \\
\hline$S-5$ & $12.70-13.00$ & $>0.30$ \\
\hline S-6 & $11.90-12.00$ & $>0.10$ \\
\hline$S-7$ & $11.20-11.60$ & $>0.40$ \\
\hline$S-9$ & $10.00-15.00$ & $>5.00$ \\
\hline$S-10$ & $11.20-14.00$ & $>2.80$ \\
\hline$S-12$ & $11.15-12.00$ & $>0.85$ \\
\hline
\end{tabular}

TABLA 10

\begin{tabular}{|c|c|c|}
\hline Fracción & Rango de Varaeión $\%$ & Valor medio \\
\hline Grava & $48-75.8$ & $61 . \overline{9}$ \\
\hline Arena & $21-50$ & $31 . \overline{8}$ \\
\hline Fincrs & $0-17$ & 63 \\
\hline
\end{tabular}


2.2.5. Sustrato Mioceno. Arcillas margosas gris verdosas ("margas azules")

Este nivel constituye el sustrato natural de la zona y se ha reconocido en los sondeos a profundidades entre los 17.90 y $18.40 \mathrm{~m}$ bajo la superficie actual, extendiéndose varios cientos de metros en profundidad (según se deduce de correlaciones regionales).

Los principales parámetros analizados se resumen a continuación:

- Los análisis granulométricos realizados reconocen un $100 \%$ de fracción fina.

. Los valores de plasticidad son elevados: $\mathrm{W}_{\mathrm{L}}=58.7, \mathrm{~W}_{\mathrm{P}}$ $=26.9, \mathrm{I}_{\mathrm{p}}=31.8$; por lo que clasificaremos el suelo como $\mathrm{CH}$.

La humedad natural medida osciló entre el 23.3 y el $26.4 \%$.

. La resistencia a compresión simple media fue de: $\mathrm{q}_{\mathrm{u}}=$ $25.7 \mathrm{t} / \mathrm{m}^{2}$. Por tanto, de consistencia muy firme, que, en profundidad, aumentará rápidamente hasta dura.

La densidad aparente fue de $=1.94-1.98 \mathrm{t} / \mathrm{m}^{3}$, quedando la densidad seca entre 1.59 y $1.62 \mathrm{t} / \mathrm{m}^{3}$.

. La cohesión y ángulo de rozamiento medidos oscilaron entre:

$\mathrm{C}^{\prime}=10-10.5 \mathrm{t} / \mathrm{m}^{2}$

$\theta^{\prime}=18.5-20^{\circ}$

. No se detecta materia orgánica ni presencia de sulfatos.

El módulo edométrico osciló entre 88.2 y $98.3 \mathrm{kp} / \mathrm{cm}^{2}$. $\left(e_{0}=0.818-0.834, C_{c}=0.11-0.12\right)$.

\section{Cimentaciones}

\subsection{Desarrollo Histórico}

El Emir al-Mu/minin Abù Ya'qùb mandó construir este alminar el año 1184, en la unión de la mezquita con la futura muralla de una nueva alcazaba. Sin embargo, tras su muerte, fue desestimado parte de este proyecto, abordándose solamente la construcción del alminar.

Muhammad b. Abì Marwàn (el granadino) se "esforzó" en la construcción de la aljama y el minarete, por orden de Abù Yùsuf, -siguiendo los designios de su padre-

El alarife Ahmad b. Basó, abrió sus cimientos -junto a la aljama- y encontró en ellos un "pozo manantial" que necesitó ser cegado con aportes de piedras y cal. Posteriormente explanó sobre el agua, hasta que se aseguraron las bases de los cimientos. A partir de este punto, es cuando se aborda la construcción del cimiento, empleándose las piedras de roca arenisca del muro del palacio próximo de Ibn 'Abbàd y de la muralla romana. Se completan los cimientos y el tramo con sillares de la base de la torre antes del año 1188 .

Las obras se paralizan, aunque no fue ésta la única vez, por cambios en el gobierno de Sevilla, hasta que en el año 1188-1189 llega Abù Bakr b. Zuhr, quien mandó continuar la obra y reparar los daños de la mezquita.

Hacia el año 1198 se culminan las obras fundamentales del alminar, invirtiéndose, por tanto, aproximadamente 14 años desde el inicio de las obras.

Los cambios más significativos en la Giralda se deben al discutido proyecto de Hernán Ruiz (Fig. 4a), que modifica el tramo superior -muy deteriorado- y construye el campanario, la azotea y los tres cuerpos superiores, quedando en una situación muy parecida a la actual (Fig. 4b). Las obras se llevaron a cabo entre 1557 y 1568 , corrigiéndose, ya en ellas, la ligera desviación que presenta la torre.

A modo anecdótico hay que indicar que la torre se ve afectada a lo largo del tiempo por infinidad de siniestros, entre los que se destacan los siguientes:

Terremotos: De ellos cabe señalar, como los de más intensidad, los acaecidos en los años i356 y 1755-1756.

Huracanes y tormentas. Los de mayor intensidad corresponden a los años: 1592, 1683 y 1685, 1836-1843, 1884 y 1887.

En la figura 5 se incluye un detalle de las principales modificaciones, a lo largo del tiempo, en la Giralda.

\subsection{Descripción de la cimentación}

Los datos suministrados por los sondeos inclinados ( $\mathrm{n}^{\circ \mathrm{s}} 3$ y 8) que atraviesan el cimiento del interior de la torre, indican la siguiente disposición sobre el basamento de la misma.

Puede afirmarse que la cimentación está constituida por capas de roca arenisca y calcarenitas, dispuestas en forma de tongadas, niveladas o rasanteadas por capas de ladrillos cerámicos recibidos con mortero. En algunos casos, esta capa llega a ser importante, convirtiéndose en una especie de conglomerado u "hormigón" de trozos de ladrillos, rocas, gravas y cal.

La roca es del tipo calcarenita-arenisca, con valores de resistencia a compresión muy variables, comolodemuestran 


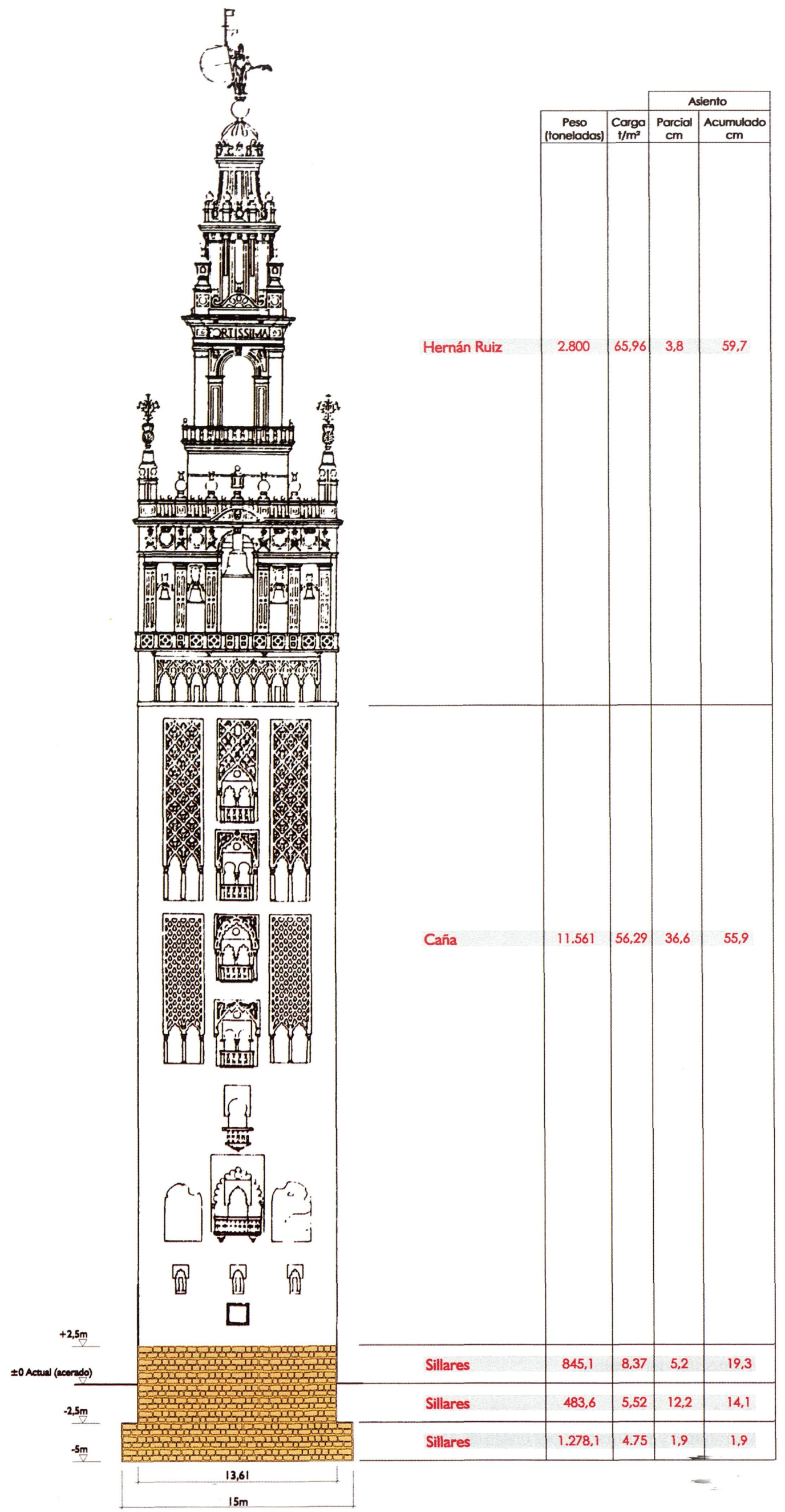




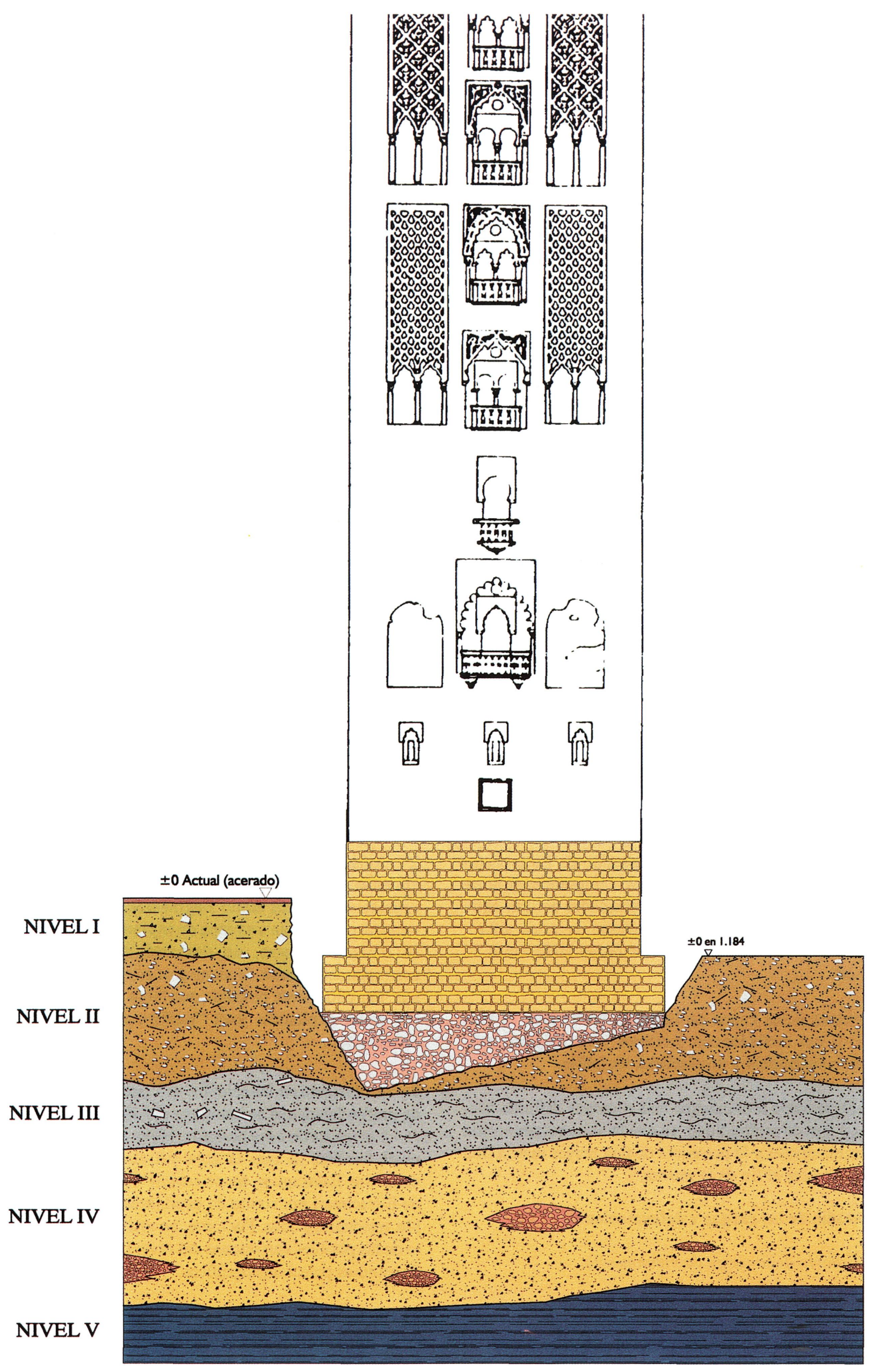

Fig. 4 (b). 


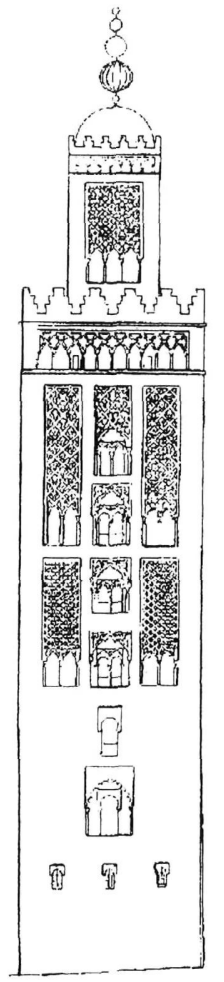

10-3-1198

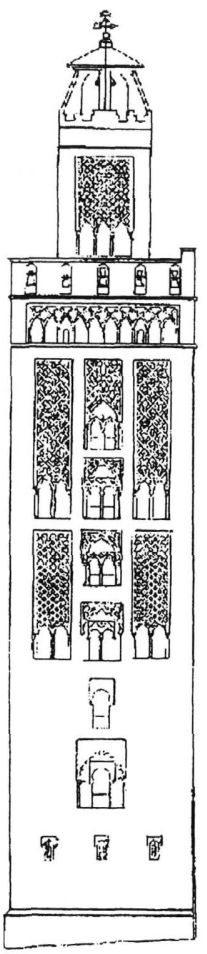

22-7-1400

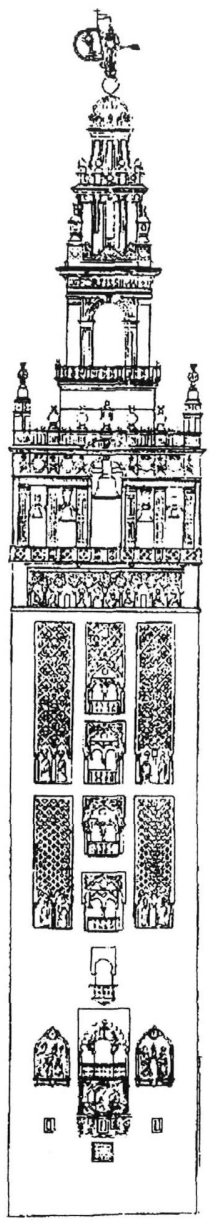

$6-10-1568$

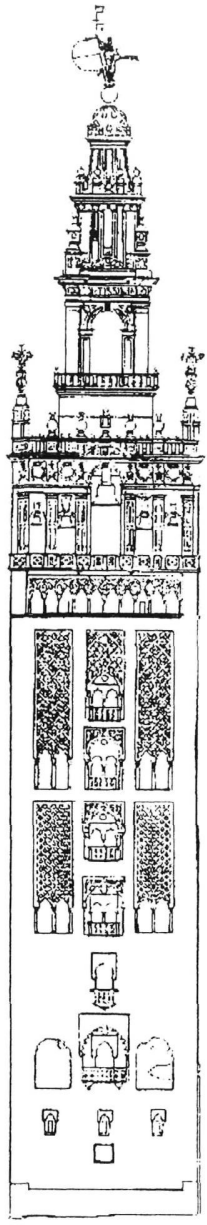

$9-7-1984$

Fig. 5

los resultados de la rotura a compresión de las probetas testigo extraídas, que dan valores comprendidos entre $30.4 \mathrm{kp} / \mathrm{cm}^{2}$ y $325.7 \mathrm{kp} / \mathrm{cm}^{2}$. Las densidades oscilan entre $1.75 \mathrm{~g} / \mathrm{cm}^{3}$ y $2.37 \mathrm{~g} / \mathrm{cm}^{3}$ La disposición de estos sillares se conforma en capas de tamaño variable, entre 25 y $60 \mathrm{~cm}$.

En cuanto a las capas de ladrillo, constituyen niveles de $10-20 \mathrm{~cm}$ recibidos con morteros y hormigones calizos de composición y propiedades variables.

Esta disposición se encuentra más o menos definida hasta los $5.0 \mathrm{~m}$ de profundidad, en que la alta contaminación de los materiales y la presencia de una mayor matriz fina parece indicar el fin de la misma.

\section{Estudio de las cargas y deformaciones}

\subsection{Cálculo de la carga de hundimiento}

\subsubsection{Hipótesis}

1.- Se ha establecido la profundidad del cimiento entre 4.9 y $5.3 \mathrm{~m}$, introduciendo en todos los cálculos el valor medio de $5.1 \mathrm{~m}$.

(c) Consejo Superior de Investigaciones Científicas Licencia Creative Commons 3.0 España (by-nc)
2.- Aunque en algunos sondeos aparecen restos de cimentación hasta los $7 / 8 \mathrm{~m}$ de profundidad, se ha considerado a éstos como fragmentos, de más o menos entidad, que forman una capa de explanación sobre la que se levanta la torre, no descartándose que puedan corresponder a restos de anteriores construcciones romanas. Por ello parece probable que fuera necesario establecer dicha explanada para "uniformar" la base de la cimentación.

3.- Se considera en los cálculos una lámina de agua a $4 \mathrm{~m}$ de profundidad, que se mantiene con ligeras oscilaciones $(+/-1 \mathrm{~m})$ en torno a esa posición. No se corresponde con el nivel freático, ya que la cota del río está por debajo de la-6m. Estas aguas, denominadas "colgadas", proceden de filtraciones por rotura de conducciones de agua potable, residuales y otras. Por tanto, no se incluye aquí el efecto de las posibles variaciones del nivel freático, deducidas de estudios paleobiogeográficos.

4.- En los cálculos que siguen se han integrado datos de 16 sondeos rotatorios realizados durante un período de más de 10 años. De ellos, 12 , se realizan junto a la Giralda, fundamentalmente en su cara Norte. 
4.1.2. Carga de hundimiento.

$P v h=q N_{q} s_{q} i_{q}+c N_{c} s_{s} i_{c}+1 / 2 \gamma B^{*} N_{\gamma} s_{\gamma} i_{\gamma}$

$\mathrm{N}_{q}, \mathrm{~N}_{\mathrm{c}}, \mathrm{N}_{\gamma}$ coeficientes de capacidad de carga.

$\mathrm{S}_{q}, \mathrm{~S}_{\mathrm{c}}, \mathrm{S}_{\gamma}$ coeficientes de forma.

$i_{q}, i_{c}, i_{\gamma}$ coeficientes de inclinación.

$\mathrm{q}$ sobrecarga de las tierras a la profundidad de la cimentación.

Se tiene

Cohesion : $\mathrm{c}^{9}=0.39 \mathrm{kp} / \mathrm{cm}^{2} ; \gamma:$ peso específico del suelo Ángulo de rozamiento interno $\theta^{\prime}=15^{\circ}$

Ancho equivalente de la cimentación $\mathrm{B}^{*}=15 \mathrm{~m}$

Desglosando, quedaría:

1) $q N_{q} s_{q} i_{q}=86 \times 3.94 \times 1.36 \times 0.96=44.24 t / m^{2}$

$q=4 \times 1.9+1.1 \times 0.9=8.6 \mathrm{t} / \mathrm{m}^{2}$

$\mathrm{N}_{\mathrm{q}}=\frac{1+\operatorname{sen} \theta}{1-\operatorname{sen} \theta} \mathrm{e}^{\eta \operatorname{tg} \theta}=3.94$

$\mathrm{s}_{\mathrm{q}}=1+\frac{\mathrm{B}^{*} \mathrm{~N}_{\mathrm{q}}}{\mathrm{L}^{*} \mathrm{~N}_{\mathrm{c}}}=1.36$

$\mathrm{i}_{\mathrm{q}}=(1-0.7 \operatorname{tg} \delta)^{3}=0.96[0.99]$

- Suponiendo $\delta=1^{\circ}$ (aunque el valor medido sea de solamente de $0^{\circ} 18^{\prime}$, resultado entre corchetes).

- También se toma $\mathrm{B}^{*}=\mathrm{L}^{*}=15 \mathrm{~m}$ (lo que supone un sobreancho a cota de cimiento de casi $1.5 \mathrm{~m}$ (13.61 a 15.0 (deducido en varios sondeos)).

2) $c N_{c} s_{s} i_{c}=3.9 \times 10.98 \times 1.36 \times 0.95=55.33 \mathrm{t} / \mathrm{m}^{2}$

$\mathrm{c}=3.9 \mathrm{t} / \mathrm{m}^{2}$

$\frac{N_{c}=N_{q}-1}{\operatorname{tg} \theta}=10.98$

$\mathrm{s}_{\mathrm{c}}=\mathrm{s}_{\mathrm{q}}=1.36$

$\mathrm{i}_{\mathrm{c}}=\frac{\mathrm{i}_{\mathrm{q}} \mathrm{N}_{\mathrm{q}}-1}{\mathrm{~N}_{\mathrm{q}}-1}=0.95$

3) $1 / 2 \mathrm{~B} \gamma \mathrm{N}_{\gamma} \mathrm{s}_{\gamma} \mathrm{i}_{\gamma}=1 / 2 \times 15 \times 1.9 \times 2.65 \times 0.60 \times 0.95=$ $=21.52 \mathrm{t} / \mathrm{m}^{2}$
$\mathrm{N}_{\gamma}=1.5\left(\mathrm{~N}_{\mathrm{q}}-1\right) \operatorname{tg} \theta=2.65$

$\mathrm{s}_{\gamma}=1-0.4 \frac{\mathrm{B}}{\mathrm{L}}=0.60$

$i_{\gamma}=(1-\operatorname{tg} \delta)^{3}=0.95$

- No se toma $\gamma_{\text {sum }}$, al poder darse el caso de que el nivel freático se sitúe por debajo, lo que deja del lado de la seguridad, aunque sí se tiene en cuenta para determinar el sobrepeso actual de las tierras (1).

Por tanto, el resultado será:

$\mathrm{PV}_{\mathrm{h}}=44.24+55.33+21.52=121.09 \mathrm{t} / \mathrm{m}^{2}$

Dado que la cohesión obtenida puede resultar ligeramente elevada para este tipo de suelo, se han realizado los cálculos utilizando un valor de cohesión de $c^{\prime}=1.5 \mathrm{t} / \mathrm{m}^{2}$, obteniéndose el siguiente resultado:

$P v_{h}=44.24+21.28+21.52=87.04 t / \mathrm{m}^{2}$

\subsubsection{Anexo para determinación de pesos}

Correlacionando con datos de informes previosy corrigiendo ligeramente a la baja el peso total para el cimiento, nos queda:

\section{Cimiento}

$\begin{array}{ll}\text { peso }_{1} & 3 \times 13.61^{2} \times 2.3=1.278 .1 \mathrm{t} \\ \text { peso }_{2} & 1 \times 14.5^{2} \times 2.3=483.6 \mathrm{t} \\ \text { peso }_{3} & 1.4 \times 16.2^{2} \times 2.3=845.1 \mathrm{t}\end{array}$

\section{$2.606 .8 \mathrm{t}$}

\section{Peso caña (Total)}

peso, $\quad 52 \times 13.61^{2} \times 2.3 \mathrm{~F}$ (hueco) $=12.161$

peso, $_{1}, 2.5 \times 13.61^{2} \times 2.3 \times \mathrm{F}=600 \mathrm{t}$

peso $_{2^{\prime}}=$ peso $_{1}-$ peso $_{1^{\prime}}=11561 \mathrm{t}$

\section{Peso total de la Giralda}

peso $_{t}=14.336 t+2.606 .8 t=16.942 t=17.000 t$

Peso $=1.7 \times 10^{4} \mathrm{t}$

\section{Carga transmitida al suelo a cota del cimiento:}

$\mathrm{Q}=1.7 \times 10^{4} \mathrm{t} / 15^{2} \mathrm{~m}^{2}=64.78 \mathrm{t} / \mathrm{m}^{2}$

$$
\mathrm{Q}=6.5 \mathrm{kp} / \mathrm{cm}^{2}
$$


4.2. Cálculo de asientos en las diferentes etapas de su construcción

\subsubsection{Hipótesis}

1. Se supone la topografia primitiva coincidiendo con el techo del nivel $2 c$, asimilable a mezclas de relleno, depósitos fluvio-aluviales recientes y otros (ver perfil).

2. Se define la capa compresible a toda la zona que queda comprendida entre la base del cimiento y el techo del nivel de gravas.

- El espesor es variable entre 6.0 y $7.5 \mathrm{~m}$, lo que explica la existencia de asientos diferenciales que inducen a una ligera inclinación de la torre con componente Sur, coincidiendo con el mayor espesor de suelos blandos compresibles, como se verá posteriormente.

- El valor medio de $h_{i}=6.95 \mathrm{~m}$ permite el cálculo del asiento referido al punto medio del estrato.

- Se considera el estrato compresible homogéneo en su conjunto y compuesto, principalmente, por arcillas y limos normalmente consolidados.

3. Se considera el asiento en la capa de gravas como inmediato y muy reducido, a tenor de su elevada compacidad.

4. Por idéntica razón y por quedar bajo la capa de gravas no se considera el asiento sobre la marga azul subyacente.

5. La sobrecarga de la torre es precedida por una descarga para la excavación del cimiento, dato éste que se ha tenido en cuenta para determinar la $\mathrm{q}_{\text {neta }}$.

- Se supone una excavación media mínima de $2.5 \mathrm{~m}$, a partir de la cota $-2 \mathrm{~m}$, tomada como superficie del terreno en este punto.

- De este modo:

- La sobrecarga de tierras en el punto medio de la capa compresible será:

. Nivel freático medio a $-4 \mathrm{~m}$ (equivalente a $-2 \mathrm{~m}$, descontando el relleno reciente).

$$
\begin{aligned}
& . h_{1}=6.95 \mathrm{~m} \\
& q=2 \times 1.9+1.1 \times 0.9+\frac{h_{1}}{2} \times 0.9=7.92 \\
& q=7.92 \mathrm{t} / \mathrm{m}^{2}
\end{aligned}
$$

- Las tierras excavadas, serán:

$$
\mathrm{q}_{\text {exc }}=1.5 \times 1.9+1 \times 0.9=3.75 \mathrm{t} / \mathrm{m}^{2}
$$

- Luego la carga neta, será:

$$
\mathrm{q}_{\text {neta }}=7.92-3.75 \mathrm{t} / \mathrm{m}^{2}=4.17 \mathrm{t} / \mathrm{m}^{2}
$$

6. No se considera el asiento inicial del relleno bajo el cimiento, ni la existencia de posibles cimentaciones más antiguas.

4.2.2. Estimación de asientos por el método edométrico.

Se han realizado hasta un total de 7 ensayos edométricos à muestras del suelo o nivel sobre el que se apoya la cimentación de la Giralda. Los datos se indican a continuación, obteniendo el valor medio como más representativo.

- Índice de poros, $\mathrm{e}$

$$
\begin{aligned}
& =\frac{0.754+0.827+0.879+0.826+0.843+0.995+0.817}{7}= \\
& =0.849
\end{aligned}
$$

- Módulo edométrico, $\mathbf{E}_{\mathrm{m}}$

$\mathrm{E}_{\mathrm{m}}=\frac{61+66+44.7+46+49.4+53.7+66}{7}=55.23 \mathrm{kp} / \mathrm{cm}^{2}$

\section{- Índice de compresión, $C_{c}$}

$C_{c}=\frac{0.12+0.17+0.17+0.17+0.183+0.166+0.17}{7}=0.164$

4.2.3. Cálculo de asientos. Desarrollo matemático

$\mathrm{S}=\frac{\mathrm{H}}{1+\mathrm{e}_{\mathrm{o}}} \mathrm{C}_{\mathrm{c}} \quad \log \left(\frac{\sigma_{\mathrm{i} 0}+\Delta \sigma_{\mathrm{i}}}{\sigma_{\mathrm{i} 0}}\right)$

Donde:

$\mathrm{S}=$ asiento en el punto medio de la capa compresible.

$\mathrm{H}=$ espesor de la capa compresible.

$\sigma_{\mathrm{i} 0}=$ carga inicial.

$\Delta \sigma_{\mathrm{i}}=$ incremento de carga.

$\mathrm{e}_{0}=$ índice de poros

$\mathrm{C}_{\mathrm{c}}=$ indice de compresión.

Siendo, en este caso:

$e_{o}=0.849 ; C_{c}=0.164 ; H=6.95 \mathrm{~m} ; \sigma_{i 0}=7.92 \mathrm{t} / \mathrm{m}^{2}$ 
Despejando:

$S=\frac{6.95}{1+0.849} 0.164\left[\log \left(\sigma_{\omega}+\Delta \sigma_{i}\right)-\log 4.17\right]$

Si no incluimos la excavación, será:

$\sigma_{i 0}=7.92 \mathrm{t} / \mathrm{m}^{2}$

$$
\mathrm{S}=0.616 \log \left(7.92+\mathrm{D} \sigma_{\mathrm{i}}\right)-0.554^{*}
$$

( En este caso se introducirá la excavación, con signo negativo, en el cálculo de $\Delta \sigma_{i}$ ).

\section{Asientos en los diferentes estadios de la construcción de la Giralda}

\subsection{Excavación}

Se procede a la excavación de la plataforma sobre la que se asienta la Giralda.

Con toda probabilidad esta excavación, independientemente de la cota desde la que se inicie, alcanza y supera la posición del nivel freático, por lo que se procede a efectuar un relleno con material heterogéneo sobre el estrato de consistencia fangosa.

Es posible que este relleno consolide parte del estrato blando, registrándose un espesor claramente superior a los $2 \mathrm{~m}$ en algunos puntos.

No se descarta la posible existencia de antiguas cimentaciones en la zona. Sin embargo, parece poco probable que el cimiento de la Giralda se extienda más allá de los $5.1 / 5.3 \mathrm{~m}$ bajo la superficie actual.

No se realizan cálculos de asientos en este punto, ya que el peso de la excavación compensa sobradamente al relleno.

\subsection{Cimientos $1^{a}$ Fase $(=2.5 \mathrm{~m})$}

Se ha considerado una plataforma aproximada de $15 \mathrm{~m}$ de lado a esta cota, en base a los sondeos S- 1 y S-2 (desplazados hasta $1.5 \mathrm{~m}$ de la vertical de la torre y que cortan el cimiento en profundidad).

- Incremento de carga: $\Delta \sigma_{i}$

$\Delta \sigma_{i}=1 \times 1.3+1.5 \times 2.3=4.75 \mathrm{t} / \mathrm{m}^{2}$

Incluyendo la excavación en este punto

$\Delta \sigma=4.75-4.17=0.58 \mathrm{t} / \mathrm{m}^{2}$

\section{- Asiento inducido}

$S=0.616 \log \left(7.92+\Delta \sigma_{i}\right)-0.554$

$$
\mathrm{S}=0.019 \mathrm{~m}=1.9 \mathrm{~cm}
$$

\subsection{Cimientos $2^{a}$ Fase $(2.6 \mathrm{~m})$}

Se considera en esta segunda etapa la terminación del cimiento, con una reducción en el lado hasta los $13.61 \mathrm{~m}$ que presenta en su tramo subaéreo.

El factor reductor de área será, por tanto:

$$
\mathrm{F}=\frac{15^{2}}{13.61^{2}}=1,21 ; \quad \mathrm{F}_{\mathrm{A}}=\frac{1}{1.21}
$$

Se supone la carga perfectamente centrada.

- Incremento de carga: $\Delta \sigma_{i}$

$\Delta \sigma_{\mathrm{i}}=0.58+\mathrm{F}_{\mathrm{A}}(2.6 \times 2.3)=5.52 \mathrm{t} / \mathrm{m}^{2}$

$$
\Delta \sigma_{i}=5.52 \mathrm{t} / \mathrm{m}^{2}
$$

- E1 asiento inducido:

$S=0.616 \log \left(7.92+\Delta \sigma_{i}\right)-0.554$

$$
\mathrm{S}=0.141 \mathrm{~m}=14.1 \mathrm{~cm}
$$

\subsection{Muro de piedra de la Giralda $(=2.5)$}

Los primeros metros presentan un muro de piedra exterior, si bien ya no es macizo como el cimiento infrayacente al existir el hueco interior de acceso a la parte superior, por lo que habrá que multiplicar por un factor corrector que, en este primer caso, será $\mathrm{F}_{\mathrm{B}}=0.6$ y para el resto de la caña será de $\mathrm{F}_{\mathrm{B}}=0.53$.

- Incremento de carga: $\Delta \sigma_{i}$

$\Delta \sigma_{\mathrm{i}}=5.52+\left(\mathrm{F}_{\mathrm{A}} \times \mathrm{F}_{\mathrm{B}} \times 2.5 \times 2.3\right)=8.37 \mathrm{t} / \mathrm{m}^{2}$

$$
\Delta \sigma_{i}=8.37 \mathrm{t} / \mathrm{m}^{2}
$$

- Asiento inducido

$S=0.616 \log \left(7.92+\Delta \sigma_{i}\right)-0.554$

$$
\mathrm{S}=0.193 \mathrm{~m}=19.3 \mathrm{~cm}
$$




\subsection{Construcción de la caña}

La construcción de la caña se produce de forma continuada, alcanzando los $49.5 \mathrm{~m}$ sobre la posición anterior.

$\mathrm{F}=\mathrm{F}_{\mathrm{A}} \times \mathrm{F}_{\mathrm{B}}=0.44$

- Incremento de carga: $\Delta \sigma_{i}$

$\Delta \sigma_{i}=8.37+(0.44 \times 49.5 \times 2.2)=56.29 \mathrm{t} / \mathrm{m}^{2}$

$$
\Delta \sigma_{i}=56.29 \mathrm{t} / \mathrm{m}^{2}
$$

para el cálculo de cada metro, tendremos:

$\Delta \sigma_{i}=\left(\Delta \sigma_{j-1}+0.968\right) t / m^{2}$

- Asiento inducido

$S=0.616 \log \left(7.92+\Delta \sigma_{i}\right)-0.554$

$$
\mathrm{S}=0.559 \mathrm{~m}=55.9 \mathrm{~cm}
$$

\subsection{Asiento máximo: estado actual}

Partiendo de los datos en peso totales de la torre y restándole a éstos el peso de la caña más el peso del cimiento, nos queda el peso del cuerpo superior:

peso $=2.175 \mathrm{t}$

- Incremento de carga: $\Delta \sigma_{i}$

$\Delta \sigma_{i}=2.175 \mathrm{t} / 15^{2} \mathrm{~m}^{2}=9.67 \mathrm{t} / \mathrm{m}^{2}$

$\Delta \sigma_{i}=56.29+9.67=65.96 \mathrm{t} / \mathrm{m}^{2}$

$$
\Delta \sigma_{i}=65.96 \mathrm{t} / \mathrm{m}^{2}
$$

- Asiento inducido

$S=0.616 \log (7.92+6596)-0.554$

$$
\mathrm{S}=0.597 \mathrm{~m}=59.7 \mathrm{~cm}
$$

Por tanto, $\mathrm{S}_{\max }=59.7 \mathrm{~cm}$ en el punto medio de la capa compresible.

5.7. Asiento diferencial con la caña completamente construida

Si se considera la variación en la capa compresible desde 6 hasta $7.5 \mathrm{~m}$, se tiene un asiento en cada punto de:
. Para $6 \mathrm{~m}$. la carga $\sigma_{i}=7.49 \mathrm{t} / \mathrm{m}^{2}$

$\mathrm{h}_{\mathrm{i}}=6.00 \mathrm{~m}$

$\mathrm{q}=2 \times 1.9+1.1 \times 0.9+\frac{\mathrm{h}_{\mathrm{i}}}{2} \times 0.9=7.49$

$\mathrm{q}=7.49 \mathrm{t} / \mathrm{m}^{2}$

$\Delta \sigma_{i}=(7.49-7.92)+56.29=56.72$

$S=\frac{6.00}{1+0.849} 0.164\left[\log \left(\sigma_{i 0}+\Delta \sigma i\right)-\log 7.49\right]$

$$
S=0.532 \log (63.35)-0.465=0.49 m
$$

. Para $7.5 \mathrm{~m}$, la carga $\sigma_{i}=8.17 \mathrm{t} / \mathrm{m}^{2}$.

$\mathrm{h}_{\mathrm{i}}=7.50 \mathrm{~m}$

$q=2 \times 1.9+1.1 \times 0.9+\frac{h}{2} \times 0.9=8.17$

$$
\mathrm{q}=8.17 \mathrm{t} / \mathrm{m}^{2}
$$

$\Delta \sigma_{1}=(8.49-7.92)+56.29=56.86$

$S=\frac{7.50}{1+0.849} 0.164\left[\log \left(\sigma_{i 0}+\Delta \sigma i\right)-\log 8.17\right]$

$$
\mathrm{S}=0.665 \log (65.03)-0.607=0.599 \mathrm{~m}
$$

Esto supone un asiento diferencial de $0.105 \mathrm{~m}$, lo que implica una distorsión de:

Distorsión $=\frac{0.105}{15.000}=0^{\circ} 24^{\prime} 03.83^{\prime \prime}$

Para una altura calculada total de $57.1 \mathrm{~m}$ desde el cimiento, supondría una desviación máxima de $0.40 \mathrm{~m}$.

El valor real máximo medido es de $25.5 \mathrm{~cm}$, a lo que habría que sumar la desviación en el cimiento, por lo que puede correlacionarse esta desviación en la Giralda con el diferente espesor de suelos blandos entre un punto y otro de la torre, siendo mayor en la zona SE. Esto coincide con la inclinación del eje de la torre, que presenta una ligera desviación que puede explicarse por la rigidez del cimiento o por lo simplificado del modelo.

A continuación se incluyen las desviaciones en las caras Este, Norte y Oeste. 
67

Informes de la Construcción, Vol. 49 n 452, noviembre/diciembre 1997

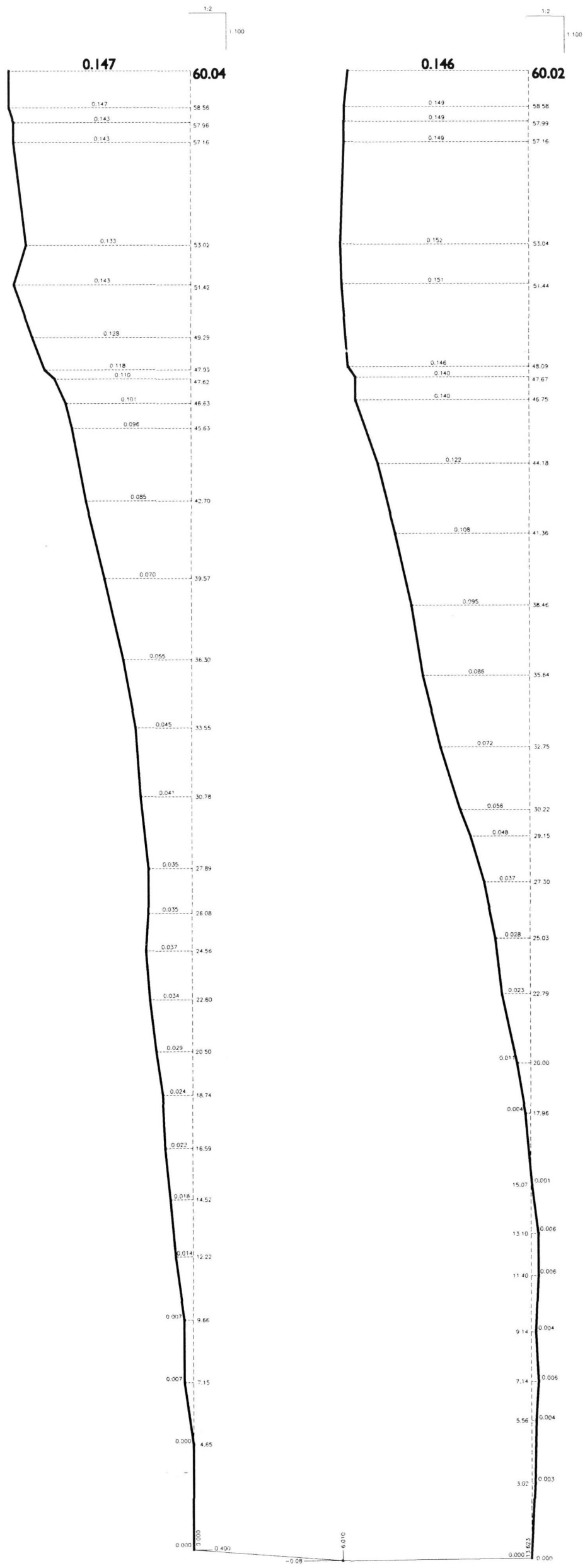

Desviaciones. Cara Este.

(c) Consejo Superior de Investigaciones Científicas

Licencia Creative Commons 3.0 España (by-nc)

http://informesdelaconstruccion.revistas.csic.es 
68

Informes de la Construcción, Vol. 49 n 452 , noviembre/diciembre 1997
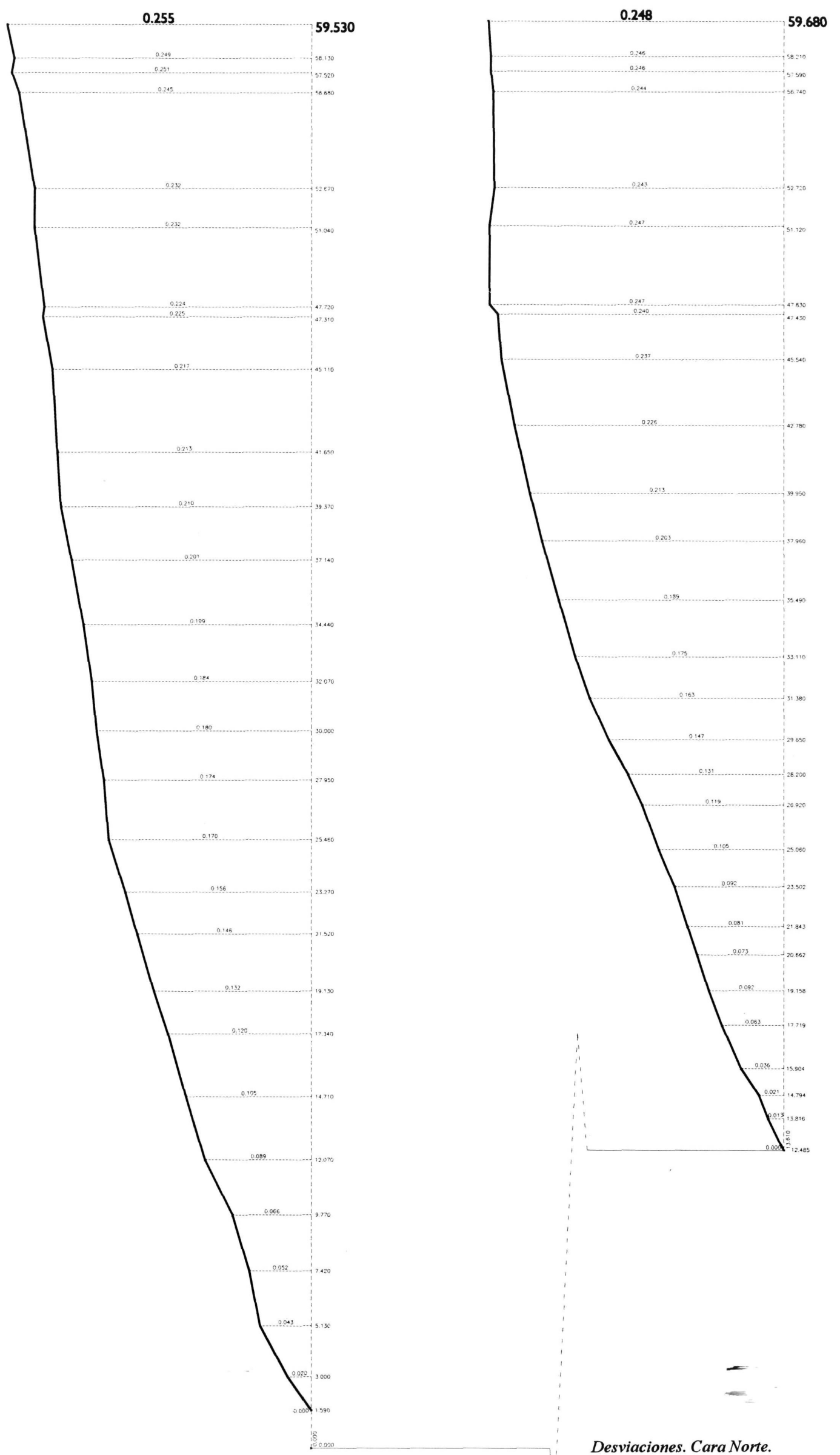

Desviaciones. Cara Norte.

(c) Consejo Superior de Investigaciones Científicas

Licencia Creative Commons 3.0 España (by-nc)

http://informesdelaconstruccion.revistas.csic.es 


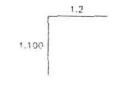

47.120

0.171

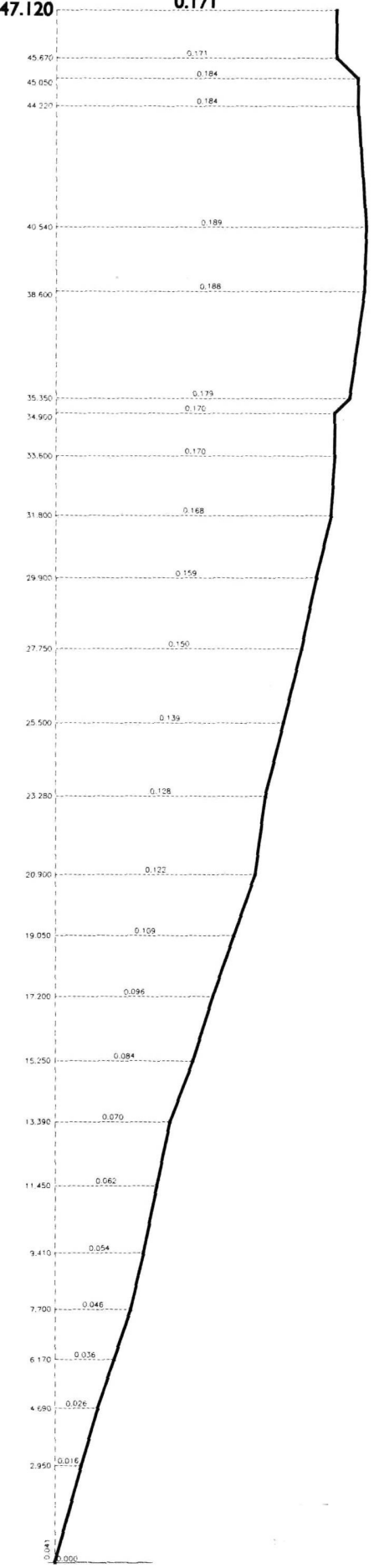

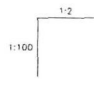

47.033

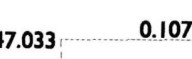

456 .107

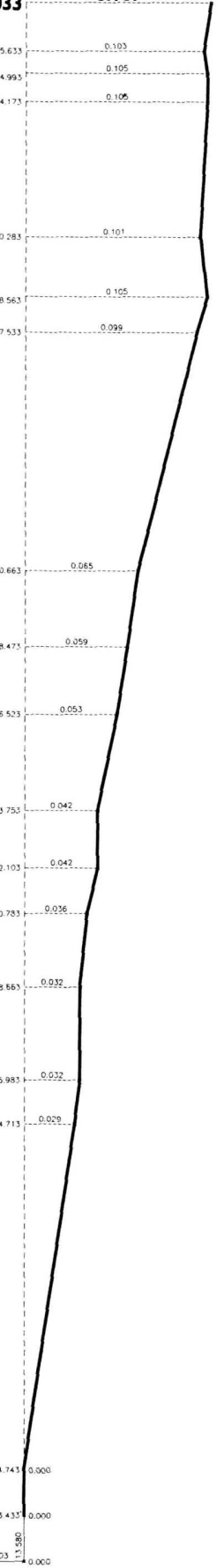




\section{Evaluación temporal del asiento en función de las cargas aplicadas para diferentes intervalos de consolidación}

A continuación se estudia el grado de consolidación que induce la parte de la obra realizada en un tiempo dado, conocida por la evolución de la obra de acuerdo con referencias históricas.

Para ello se ha deducido el valor de $\left[\mathrm{t}_{\mathrm{V}_{\text {medo }}}\right.$ (Coeficiente de consolidación)] en lacurva de consolidación de los diferentes edómetros realizados, resultando:

$$
\begin{aligned}
C_{v} & =\frac{1.35 \times 10^{-3}+2.33 \times 10^{-4}+4.2 \times 10^{-4}}{3}= \\
& =6.67667 \times 10^{-4} \mathrm{~cm}^{2} / \mathrm{s}
\end{aligned}
$$

$$
\mathrm{C}_{\mathrm{v}}=6.677 \times 10^{-4} \mathrm{~cm}^{2} / \mathrm{s}
$$

Como quiera que:

$\mathrm{T}=\frac{\mathrm{C}_{\mathrm{v}} \mathrm{t}}{\mathrm{H}^{2}} ; \quad \mathrm{H}=\frac{\mathrm{H}_{\mathrm{i}}}{2}=\frac{695}{2}=347.5$

Donde:

$\mathrm{T}=$ coeficiente de tiempo

$\mathrm{H}=$ espesor de la capa

$\mathrm{t}=$ tiempo de consolidación

y que $\quad \mathrm{U}=\left(\frac{\mathrm{T}^{3}}{\mathrm{~T}^{3}+0.5}\right)^{1 / 6}$

valores que se encuentran tabulados.

siendo $\mathrm{U}=$ grado de consolidación.

De este modo, tenemos:

$\mathrm{U}=0.50(50 \%$ consolidación $) \mathrm{T}=0.197$

Despejando:

$\mathrm{t}=\frac{\mathrm{TH}^{2}}{\mathrm{C}_{\mathrm{v}}}$

\subsection{A la terminación del cimiento}

Es de suponer que se empleó en esta tarea 3 años, desde 1184 a 1187.

$\mathrm{t}=3$ años $=64.608 .000 \mathrm{~s}$.

$\mathrm{T}=\frac{\mathrm{tC}_{\mathrm{v}}}{\mathrm{H}^{2}}$

(c) Consejo Superior de Investigaciones Científicas Licencia Creative Commons 3.0 España (by-nc)
Luego $\mathrm{T}=0.523 \mathrm{y}$, por tanto, $\mathrm{U}=0.77(77 \%$ de consolidación).

En el cuadro 1 relacionamos $\mathrm{U}, \mathrm{S}$ y $\mathrm{T}$ con el tiempo.

El asiento total era de $14.1 \times 0.77=10.86 \mathrm{~cm}$.

\subsection{Primera parte de la caña}

La primera fase de la obra culmina con los 2.5 primeros metros de altura de la caña, realizada en sillares de arenisca, en lo que se invierten 4-5 años (incluidos los de la fase anterior).

$\mathrm{t}=5$ años $=157.680 .000 \mathrm{~s}($ Cuadro 2$)$.

Para 5 años

$\mathrm{T}=0.871$

$\mathrm{U}=\left(\frac{\mathrm{T}^{3}}{\mathrm{~T}^{3}+0.5}\right)^{1 / 6}=0.91(91 \%$ de consolidación $)$

$\mathrm{S}=0.91 \times 19.3=17.56 \mathrm{~cm}$

De estos 17.56 , los primeros 10.86 se deben a la carga inicial y sólo 6.7 a estos $2.5 \mathrm{~m}$ construidos y al paso de 2 años o más.

\subsection{Para toda la caña}

$\mathrm{t}=14$ años $=441.504 .000 \mathrm{~s}$

$\mathrm{T}=2.44 ; \quad \mathrm{U}=0.99$ (99\% de consolidación) (Cuadro 3).

El asiento se aproxima al valor de $55.3 \mathrm{~cm}$, de los cuales 17.56 son anteriores a la construcción de la caña y sólo 37.74 se deben a la construcción de la misma.

\subsection{Situación actual}

Comprende un incremento de asiento, debido a la carga que introduce la ampliación de Hernán Ruiz de $59.7 \mathrm{~cm}$, de los cuales sólo $4.4 \mathrm{~cm}$ se deben a esta ampliación, con un grado de consolidación próximo al $100 \%$.

En la gráfica 1 se refleja la curva de asientos total resumen de los asientos obtenidos para cada periodo, reajustándolos al asiento total.

\section{Conclusiones}

Se resumen aqui los aspectos esenciales tratados a lo largo del presente trabajo y al que se remite para una explicación más detallada. 
CUADRO 1

\begin{tabular}{|c|c|c|c|}
\hline Grado de Consolidación & $\mathrm{T}$ & $\mathrm{t}$ (años y días) & Asiento $(\mathrm{cm})$ \\
\hline $10 \%$ & 0.007 & 14.96 dias & 1.41 \\
\hline $50 \%$ & 0.197 & 1 año y 56.13 días & 7.05 \\
\hline $99 \%$ & 2.000 & 11 años y 260.51 días & 13.96 \\
\hline
\end{tabular}

CUADRO 2

\begin{tabular}{|c|c|c|c|}
\hline Grado de Consolidación & $\mathrm{T}$ & $\mathrm{t}$ (años y días) & Asiento $(\mathrm{cm})$ \\
\hline $10 \%$ & 0.007 & 14.96 días & 1.93 \\
\hline $50 \%$ & 0.197 & 1 año y 56.13 días & 9.65 \\
\hline $99 \%$ & 2.000 & 11 años y 260.51 días & 19.11 \\
\hline
\end{tabular}

CUADRO 3

\begin{tabular}{|c|c|c|c|}
\hline Grado de Consolidación & $\mathrm{T}$ & $\mathrm{t}$ (años y días) & Asiento $(\mathrm{cm})$ \\
\hline $10 \%$ & 0.007 & 14.96 días & 5.59 \\
\hline $50 \%$ & 0.197 & 1 año y 56.13 días & 27.95 \\
\hline $99 \%$ & 2.000 & 11 años y 260.51 dias & 55.34 \\
\hline
\end{tabular}

GRÁFICA 1

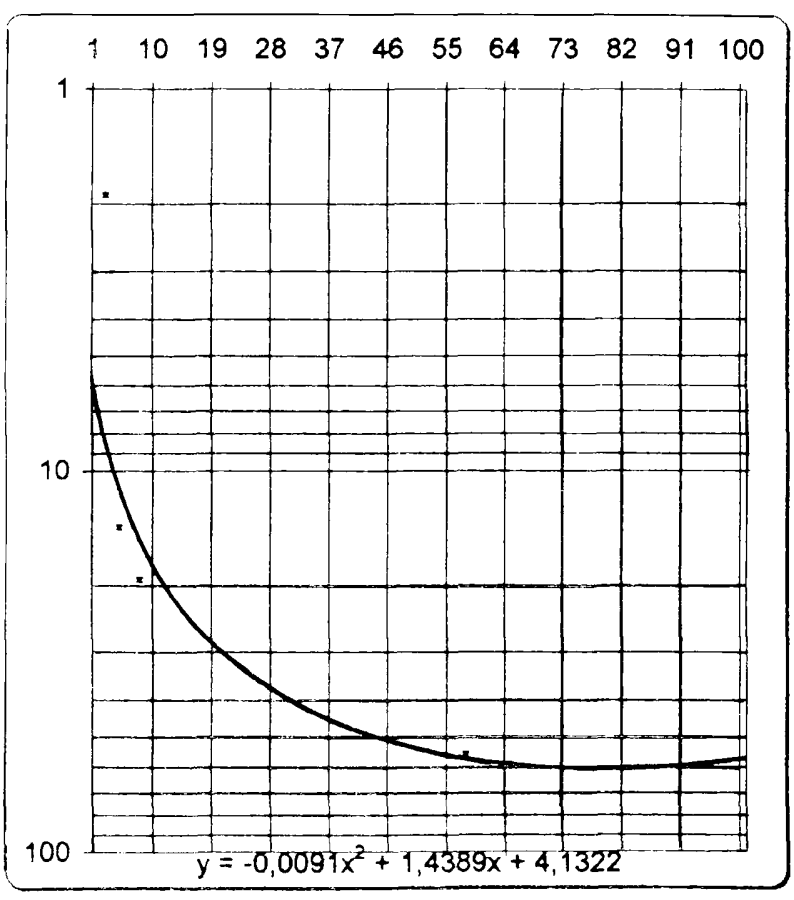

- La investigación realizada comprende un total de 14 sondeos rotatorios, ubicados, principalmente, en una lineación N-S que corta la planta de la Giralda en su zona central. También se sondea en la cara Este de la misma, habiéndose tenido en cuenta, además, otros sondeos realizados en el interior de la Catedral.
De estos sondeos, tres se ejecutan inclinados, para reconocer el cimiento en profundidad.

El resto de las investigación consistió en la recopilación de información sobre los principales sucesos que afectan a la torre, desde su construcción a la actualidad.

- Una primera conclusión que se deduce de la observación de los perfiles de sondeos es que la estratigrafía de la zona se aparta ligeramente de la serie fluvial tipo que existe en Sevilla. Aquí, el espesor de relleno y/o sedimento aluvial reciente es anormalmente elevado y descansa, discordantemente, sobre un estrato arcilloso grisáceo plástico (inédito en otras zonas). Esta capa puede asociarse con facies semipantanosas o de charca.

A partir de unos $12 \mathrm{~m}$ se reconoce el tramo basal de la serie fluvial típica, con un espesor medio de $6 \mathrm{~m}$ de arenas y gravas arenosas silíceas, con episodios conglomeráticos y bolos hacia la base.

Subyacente aparece el sustrato margoso mioceno ("Margas azules").

- La excavación para la ejecución del cimiento de la Giralda alcanzó y superó la posición del nivel freático en la zona en aquel tiempo, como se deduce de las referencias a un "pozo manantial" que necesitó cegarse y explanar sobre él, posteriormente, el cimiento.

De los datos recogidos en sondeos se deduce un plano de cimiento a-5. 1 m desde la superficie actual, siendo frecuen- 
te encontrar restos que provendrian del relleno y posterior explanada. Esto sería difícil de explicar si se tiene en cuenta la situación del nivel freático actual (entre - 3 y $-4 \mathrm{~m}$ ). No obstante, existen estudios paleobiogeográficos que sitúan el nivel freático más bajo del nivel actual en esa época (1184), lo que permitiría justificar la posibilidad de realizar una excavación tan profunda para la transmisividad de los niveles superiores reconocidos en la estratigrafia de la zona.

Además hemos de indicar que la excavación se produce rápidamente (pocos meses), dada la naturaleza de los materiales.

- Con este modelo geomecánico se ha calculado la carga de hundimiento bajo la torre, para un cimiento de planta cuadrada de unos $15 \mathrm{~m}$ de lado, a 5.1 de profundidad:

$\mathrm{Pv}_{\mathrm{h}}=121.09 \mathrm{t} / \mathrm{m}^{2}$

Dado que la $P_{\text {trmsmisble }}=16.942 \mathrm{t} / \mathrm{m}^{2}$, se obtiene un factor de seguridad frente a hundimiento bastante bajo

$\mathrm{F}=1.61$

- Se ha realizado un cálculo aproximado de los asientos en las diferentes etapas, desde su construcción, por el método edométrico (Cuadro 4)

. Se ha considerado el estrato compresible homogéneo y regular.

. Se desprecia el asiento en gravas y margas miocenas.

El incremento de carga resultante por la construcción de la Giralda es de $6.6 \mathrm{kp} / \mathrm{cm}^{2}$.

El asiento durante la construcción de la caña fue:

$$
S=0.616 \log \left(7.92-\Delta \sigma_{i}\right)-0.554
$$

siendo:

$\Delta \sigma_{i}=\Delta \sigma_{t-1}+0.968 \mathrm{t} / \mathrm{m}^{2}$ cada metro
- Si se considera el estrato compresible no uniforme, de acuerdo con su espesor real (variable entre 6 y $7.5 \mathrm{~m}$ en sección N-S), se obtiene un asiento diferencial de $0.105 \mathrm{~m}$.

\section{Esta diferencia de asientos provoca una distorsión de $0^{\circ} 24^{\prime}$}

Es decir, que se calcula una inclinación teórica de $0^{\circ} 24^{\prime}$ componente Sur para la torre, lo que supondría, al final de la caña, una desviación máxima aproximada de 0.4 . Se ha comprobado -mediante topografía- que existe una desviación de $0.255 \mathrm{~m}$ desde el pie de la torre (Fig. 6). Si a este valor se suma el correspondiente al cimiento ( y que proporcionalmente es más pesado) se obtiene un resultado muy próximo al teórico, que permite validar el modelo.

- Finalmente, se estudia la evolución temporal de los asientos, concluyéndose un alto grado de consolidación desde, prácticamente, la finalización de la obra. Esto conlleva una mejora de la capacidad portante del suelo

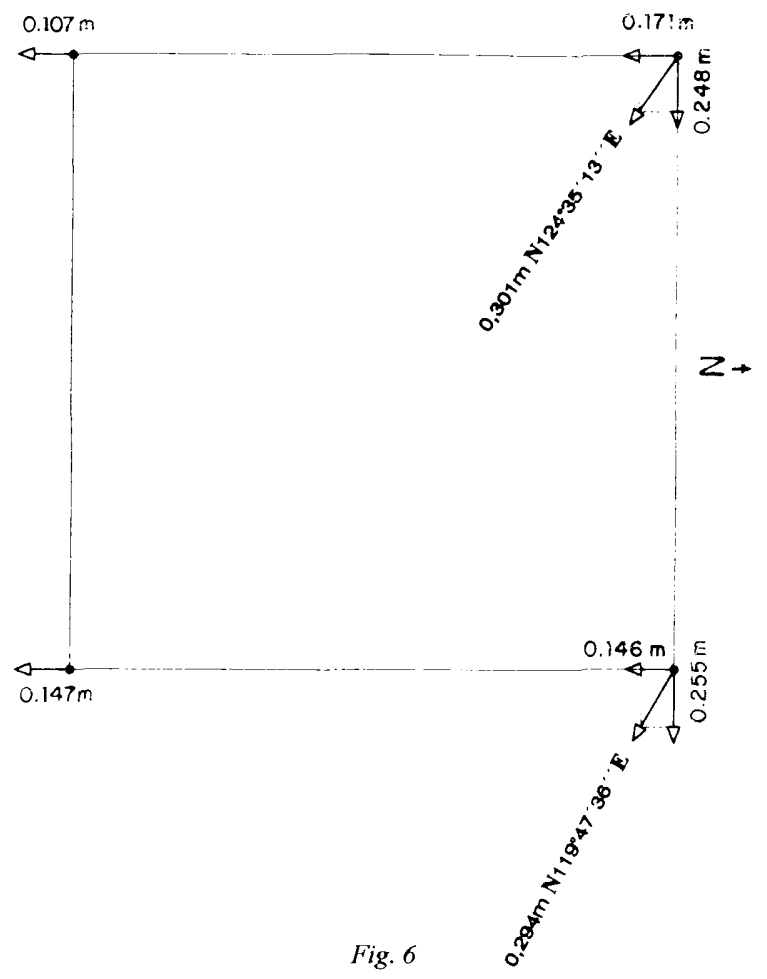

CUADRO 4

\begin{tabular}{|l|c|c|}
\hline \multicolumn{1}{|c|}{ PERIODO } & $\begin{array}{c}\text { INCREMENTO } \\
\text { DE CARGA }\end{array}$ & $\begin{array}{c}\text { ASIENTO } \\
\text { TOTAL }\end{array}$ \\
\hline Tras los $2.5 \mathrm{~m}$ primeros de cimiento (sillares de piedra) & $0.58 \mathrm{t} / \mathrm{m}^{2}$ & $1.9 \mathrm{~cm}$ \\
\hline Al terminar el cimiento (sillares de piedra con un espesor de $5 \mathrm{~m}$ en total) & $5.52 \mathrm{t} / \mathrm{m}^{2}$ & $14.1 \mathrm{~cm}$ \\
\hline Tras el tramo basal de la caña (sillares de piedra) & $8.37 \mathrm{t}^{2} \mathrm{~m}^{2}$ & $19.3 \mathrm{~cm}$ \\
\hline Al tinalizar la caña (fábrica de ladrillo) & $56.29 \mathrm{t}^{2} \mathrm{~m}^{2}$ & $55.9 \mathrm{~cm}$ \\
\hline $\begin{array}{l}\text { Tras el proyecto de Hernán Ruiz ( } \approx \text { Estado actual) (campanario, azotea } \\
\text { Giraldillo) }\end{array}$ & $65.96 \mathrm{t}^{2} \mathrm{~m}^{2}$ & $59.7 \mathrm{~cm}$ \\
\hline
\end{tabular}


por precarga, una vez que se disipa el aumento de presiones intersticiales por el edificio. Este proceso es conocido como densificación del suelo (asiento) y conduce a un incremento de la resistencia al corte sin drenaje, módulo de compresibilidad y resistencia a la penetración. Por contra, se produce una disminución de la constante de permeabilidad, el índice de poros y el contenido en agua del suelo.
(1) Con anterioridad a estetrabajo estaba extendida la creencia de que la cimentación de la Giralda se extendía por una amplia zona alrededor de la torre. Esta hipótesis se basaba en la existencia de una pavimentación, constituida por sillares de piedra arenisca idénticos a los que conforman la cimentación. Por esta razón, el número de sondeos es mayor del habitual, para determinar con exactitud las dimensiones de la cimentación.
(2) Las excavaciones arqueológicas realizadas en el verano de 1997 y en el mes de febrero de 1998, han puesto en evidencia la existencia de una losa o lecho, conformada por argamasa, sillares, hiladas de ladrillo, piedras sueltas, etc., con lo que el sobreancho dado es el que corresponde al plano de apoyo.

\section{Publicaciones del Instituto Eduardo Torroja-CSIC}

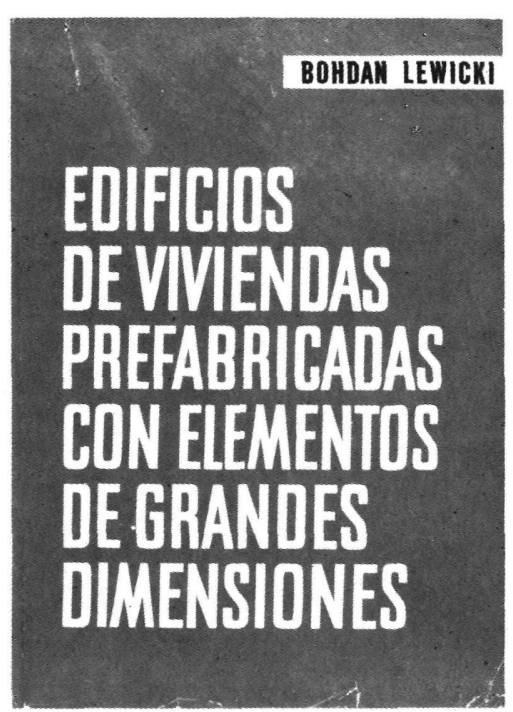

\section{Bohdan Lewicki}

Este libro trata de los problemas relativos a la construcción de los edificios de viviendas o publicos realizados con elementos prefabricados de grandes dimensiones. Se han estudiado los problemas de arriostramiento, asi como los que plantea la resistencia de los elementos y de la estructura; se han examinado las cuestiones de orden higrotérmico, acústico y de resistencia al fuego; también se ha profundizado en el estudio de la estanquidad de los muros exteriores y de las juntas.

La obra incluye numerosas ilustraciones que dan detalles de diversas soluciones, asi como ejemplos de cálculo, tablas de valores numéricos, diagramas y ábacos.

Un volumen encuadernado en tela, de $24 \times 17 \mathrm{~cm}$, compuesto de 616 págs.

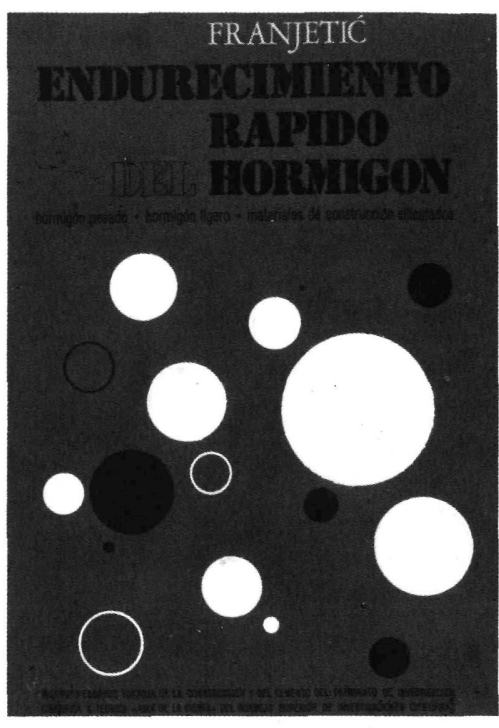

Zorislav Franjetić

En la obra de Franjetić se expone de una forma minuciosa, ordenada y sistemática, todo un cuerpo de doctrina que reúne el conocimiento actual sobre el endurecimiento rápido del hormigón. Parte el autor de los principios básicos y llega a las últimas consecuencias y realidades técnicas y economicas.

Es una obra de consulta, tanto para el investigador sobre la materia, como para el proyectista y el realizador y montador de plantas e instalaciones y equipos de curado y endurecimiento rápido.

Un volumen encuadernado en cartóné, de $17 \times 24,5 \mathrm{~cm}$, compuesto de 385 págs. 110 figuras y 10 tablas.

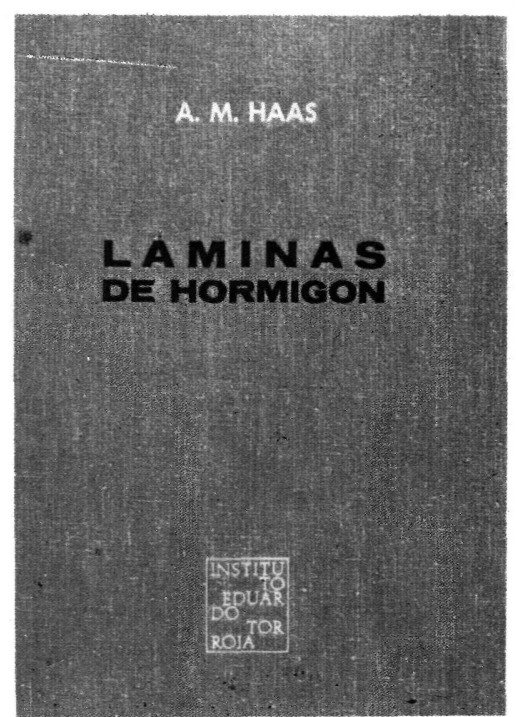

\section{A. M. Haas}

Al escribir este libro el autor intentó poner a disposición de los estudiantes y de los ingenieros unos conocimientos prácticos, adecuados para servir de guía en el diseño y construcción de láminas delgadas de hormigón.

El autor está convencido de que el éxito en el diseño de una lámina exige, por parte del proyectista, un examen de las tres fases por las que pasa la materialización de la lámina: el diseño, el análisis estructural y la construcción de la estructura.

Un volumen encuadernado en tela, de $17 \times 24,5 \mathrm{~cm}$, compuesto de 420 págs., 141 figuras, 22 fotografias y 6 tablas. 